\title{
Infiltration Heat Recovery in Building Walls: Computational Fluid Dynamics Investigations Results
}

\author{
Marc O. Abadie, Elizabeth U. Finlayson and \\ Ashok J. Gadgil \\ Lawrence Berkeley National Laboratory \\ Environmental Energy Technologies Division \\ Indoor Environment Department \\ Berkeley, CA 94720
}

August 2002

This work was supported by the Assistant Secretary for Energy Efficiency and Renewable Energy, Office of Building Technology, of the U.S. Department of Energy under Contract No. DE-AC03-76SF00098. 


\begin{abstract}
Conventional calculations of heating (and cooling) loads for buildings assume that conduction heat loss (or gain) through walls is independent of air infiltration heat loss (or gain). During passage through the building envelope, infiltrating air substantially exchanges heat with wall insulation leading to partial recovery of heat conducted through the wall. The Infiltration Heat Recovery (IHR) factor was introduced to quantify the heat recovery and correct the conventional calculations.

In this study, Computational Fluid Dynamics was used to calculate infiltration heat recovery under a range of idealized conditions, specifically to understand factors that influence it, and assess its significance in building heat load calculations. This study shows for the first time the important effect of the external boundary layers on conduction and infiltration heat loads.

Results show (under the idealized conditions studied here) that (1) the interior details of the wall encountered in the leakage path (i.e., insulated or empty walls) do not greatly influence the IHR, the overall relative location of the cracks (i.e., inlet and outlet locations on the wall) has the largest influence on the IHR magnitude, (2) external boundary layers on the walls substantially contribute to IHR and (3) the relative error in heat load calculations resulting from the use of the conventional calculational method (i.e., ignoring IHR) is between $3 \%$ and $13 \%$ for infiltrating flows typically found in residential buildings.
\end{abstract}




\section{Contents}

1. INTRODUCTION.....................................................................................................

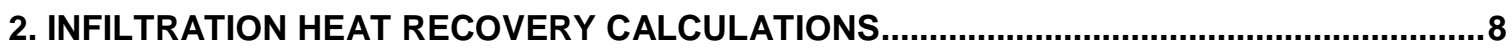

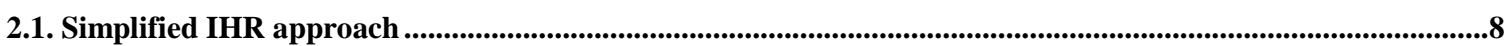

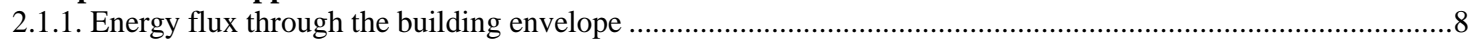

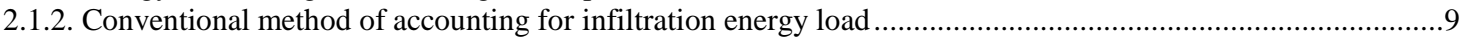

2.1.3. Infiltration Heat Recovery (IHR) calculation of infiltration load ....................................................

2.2. Combining IHR calculations for Infiltrating and Exfiltrating Walls .....................................................................11

3. DETAILS OF CFD INPUT FOR THE SIMULATED WALLS............................................13

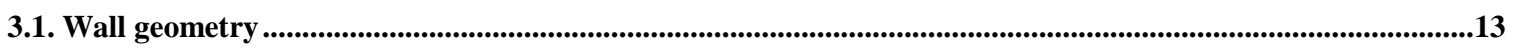

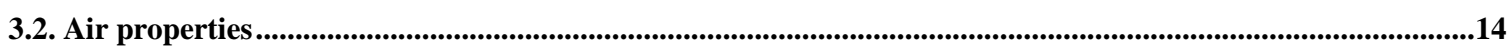

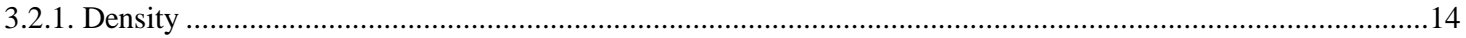

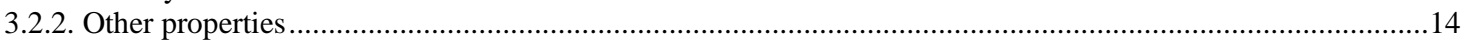

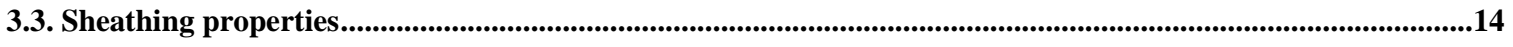

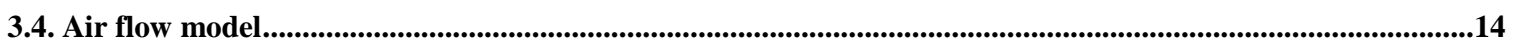

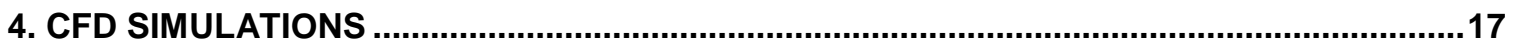

4.1. Determination of the conduction heat flux without infiltration .................................................................19

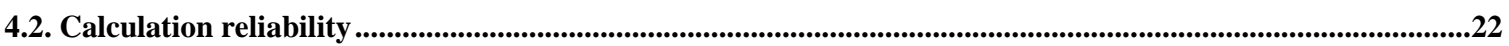

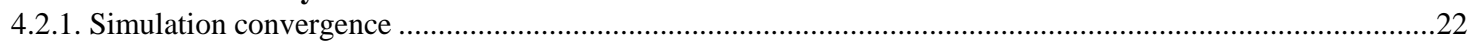

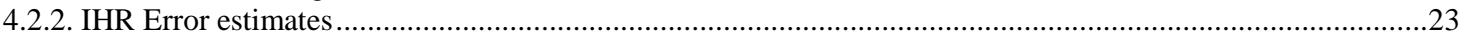

5. RESULTS.........................................................................................................26

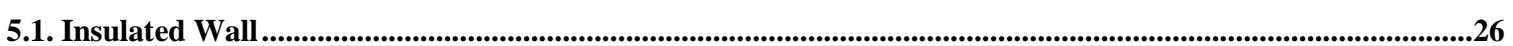

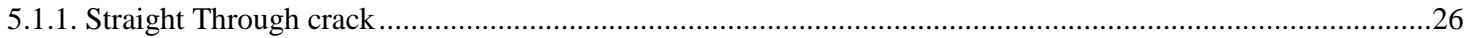

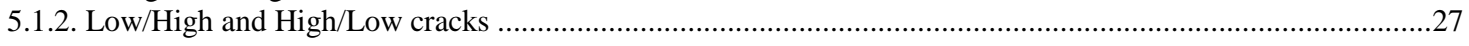

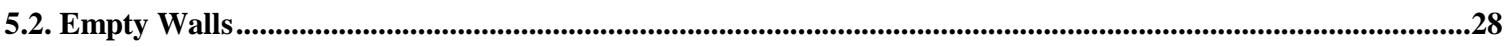

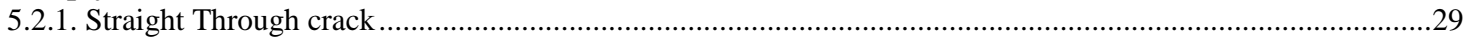

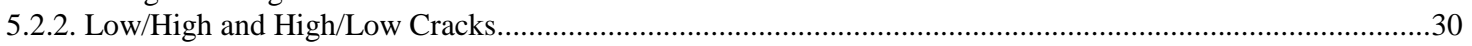

5.3. Conclusion and future developments ..........................................................................................................................31

6. ILLUSTRATIVE APPLICATIONS........................................................................32

6.1. Combining One Infiltrating and One Exfiltrating Wall to Obtain a whole building IRH value........................32

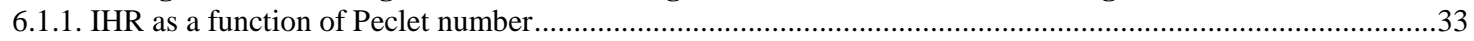

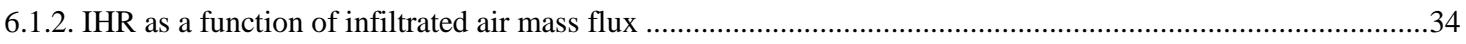

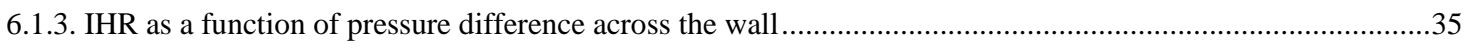

6.2. Combining One Infiltrating and Three Exfiltrating Walls to Obtain a whole building IRH value...................36

6.3. Comparison between the conventional and actual total energy load.................................................................38

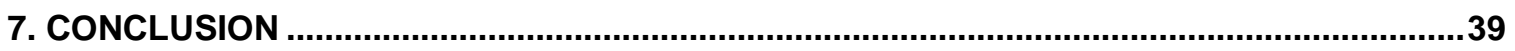

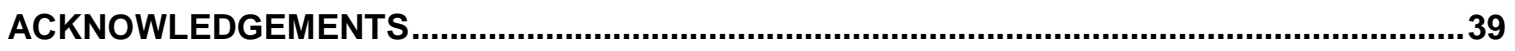


REFERENCES ............................................................................................................

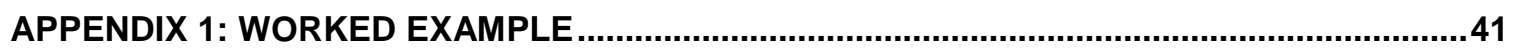




\section{Introduction}

Conventional heating (and cooling) calculations for buildings assume that conduction heat loss (or gain) is independent of air infiltration heat loss (or gain), and that the heating (or cooling) load is the direct summation of these losses. The small number of studies in the literature regarding the influence of infiltration (Caffey (1979), Persily (1982), Sherman and Matson (1993) and NIST (1996)) all conclude that the combined effect of conduction and air leakage is substantially smaller than that predicted with conventional calculations.

Considering the complex analysis required to determine the combined effects of conduction and air leakage and the need to provide a simple engineering calculation procedure that can be used by practitioners, Sherman et al $(2000,2001)$ proposed that the conventional method can be corrected by introducing only one coefficient: the infiltration heat recovery (IHR).

The present study aims to calculate the infiltration heat recovery (IHR) using Computational Fluid Dynamics (CFD); specifically to understand factors that influence it, and assess its significance in building heat load calculations.

As shown by Sherman and Walker (2001), the intermixing between the external boundary layers and the infiltrating air could play a role in the heat recovery. In order to test this assumption, we studied two configurations: in the first, a fixed temperature boundary condition is directly applied to the wall surface and in the second, a fixed temperature boundary is defined in air, some distance away from the wall, and the exterior surface flow field is modeled to include the boundary layers.

Each wall comprises two solid layers (e.g., gypsum wallboard or plywood) with space between the layers that can be filled with insulation or left empty. The two solid layers have one crack each. Air enters the wall cavity through a crack in one solid layer, traverses the space between the two layers, and leaves the wall through a crack in the opposite layer i.e. the flow is unidirectional. Six configurations of crack location and insulation were selected to cover a wide range of potential IHR values. Three cases had insulation-filled walls and three had empty wall cavities. These walls have three different positions of the inlet and outlet cracks: straight through cracks, low inlet/high outlet and high inlet/low outlet. The CFD modeling included a study of convergence criteria and grid geometry. In addition, uncertainties in predicted IHR values were calculated based on uncertainties in the CFD modeling and the calculation procedure used to calculate the IHR.

The IHR results for the infiltrating and exfiltrating walls are presented in terms of the ratio of heat transfer due to air flow to the heat transfer due to conduction: the Peclet number. The results for infiltrating and exfiltrating walls were combined to determine the overall IHR for a whole building that is required to perform energy use calculations. 


\section{Infiltration Heat Recovery Calculations}

\subsection{Simplified IHR approach}

\subsubsection{Energy flux through the building envelope}

In a real building there are many air flow paths through the envelope, all with different geometries and air flows. In addition, the conduction heat transfer changes greatly depending on whether the envelope section of interest is an insulated wall cavity, a window, a stud, etc. All these flow paths and conduction differences are unknowable in any practical sense for a real building. Therefore, in this study, we took a simplified approach in which all the air flow and conduction are effectively grouped together into single terms. Although the heat and mass flows in real envelopes are three-dimensional we used a two-dimensional model for simplicity of analysis. This two-dimensional approach offers significant advantages by simplifying CFD coding and reducing execution time for each simulation as well as allowing the use of simplified analytical calculations. This idealized approach allows the development of simple algorithms for predicting IHR and allows a systematic approach for the CFD modeling such that the effect of different parameters is easily observed. Two modes of heat transfer through the building envelope are considered: conduction through walls and convection heat transfer between the infiltrating air and the insulation and solid layers. Figure 1 illustrates these two heat-transfer modes and the physical layout for the simplified CFD model. The impact of solar as well as longwave infrared radiation on heat loads is not taken into account in this study.

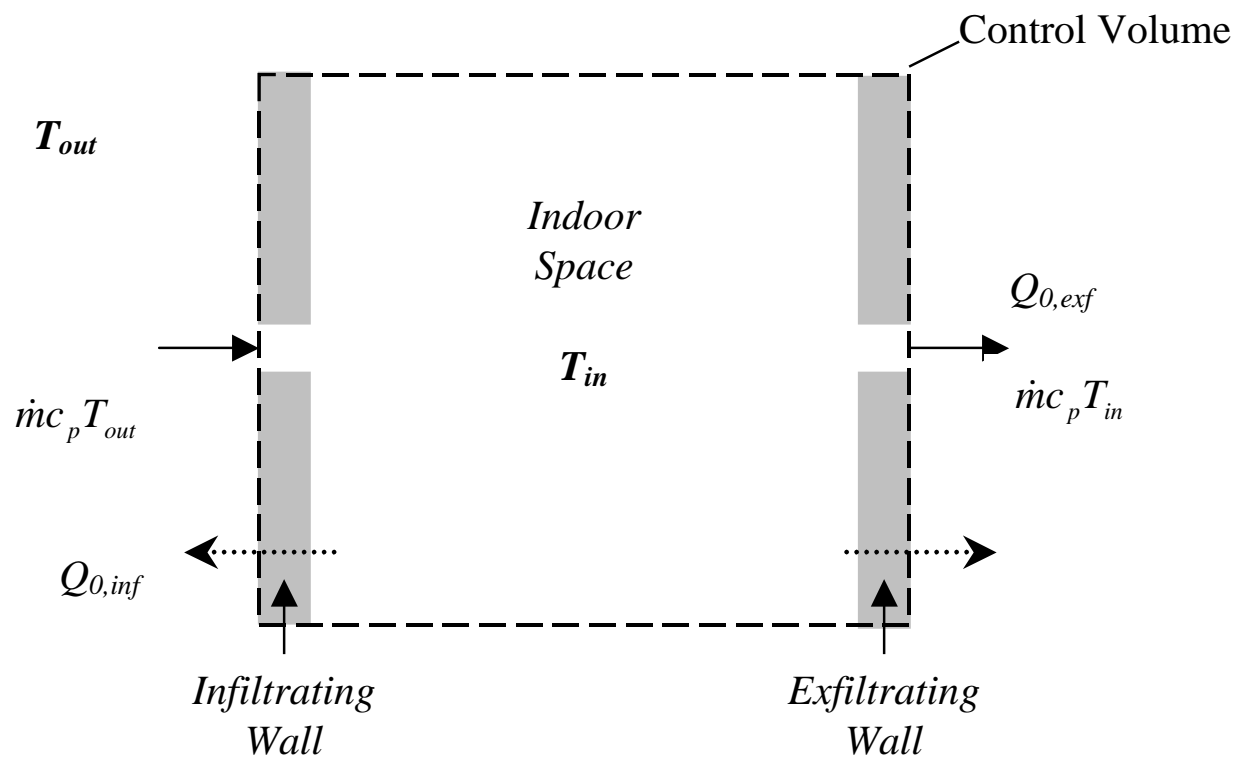

Figure 1: Cross section of a generic building envelope showing the conventional conduction and infiltration energy load terms. 


\subsubsection{Conventional method of accounting for infiltration energy load}

In the absence of infiltration through the building envelope, the heat load on the building is purely from conduction, denoted as $Q_{0}=Q_{0, \text { inf }}+Q_{0, \text { exf }}$. When there is infiltration, the total heat load using conventional methods, $Q_{c}$, is determined by adding a simple convective term to the energy balance for the building that is based on the leakage rate and the indoor and outdoor air temperatures:

$$
Q_{C}=Q_{0}+\dot{m} c_{p}\left(T_{\text {in }}-T_{\text {out }}\right)
$$

where $Q_{0}$ is the wall heat transfer by conduction without infiltration, $\dot{m}$ is the air mass flux through the walls, $c_{p}$ is the air heat capacity and $T_{i n}$ and $T_{\text {out }}$ are the indoor and outdoor temperatures respectively. This relationship assumes there is no interaction (heat transfer, moisture deposition, etc.) between the leaking air and the building walls.

\subsubsection{Infiltration Heat Recovery (IHR) calculation of infiltration load}

In reality, there is heat transfer between the solid material within the building walls and leaking air as the leaking air crosses the wall. The result is that the actual values for the conduction, the infiltration and thus the total loads (illustrated in Figure 2) are different from those predicted by the conventional method. As air penetrates the wall, heat transfer with wall material changes its temperature. Outside air enters the infiltrating wall at temperature $T_{l}$, temperature warmer than $T_{\text {out }}$ because of the conduction heat transfer from the wall. By the time it enters the room it will be at some other temperature, which we defined as $T_{2}$. Similarly, air enters the exfiltration wall at temperature $T_{3}$, temperature cooler than $T_{i n}$, and exits this wall at temperature $T_{4}$. We show the revised heat loads in equation 2 .

$$
Q=Q_{0}{ }^{*}+\dot{m} c_{p}\left(T_{4}-T_{1}\right)
$$

where $Q$ is the corrected value for the total heat load, $Q_{0}{ }^{*}$ is sum of the infiltrating and exfiltrating wall heat transfer by conduction (note that this is NOT the same as the conduction without air flow), and $T_{1}$ and $T_{4}$ are the inlet and outlet temperatures respectively (again note that in general $T_{2}$ will not equal $T_{\text {out }}$ and $T_{4}$ will not equal $T_{\text {in }}$ ). 


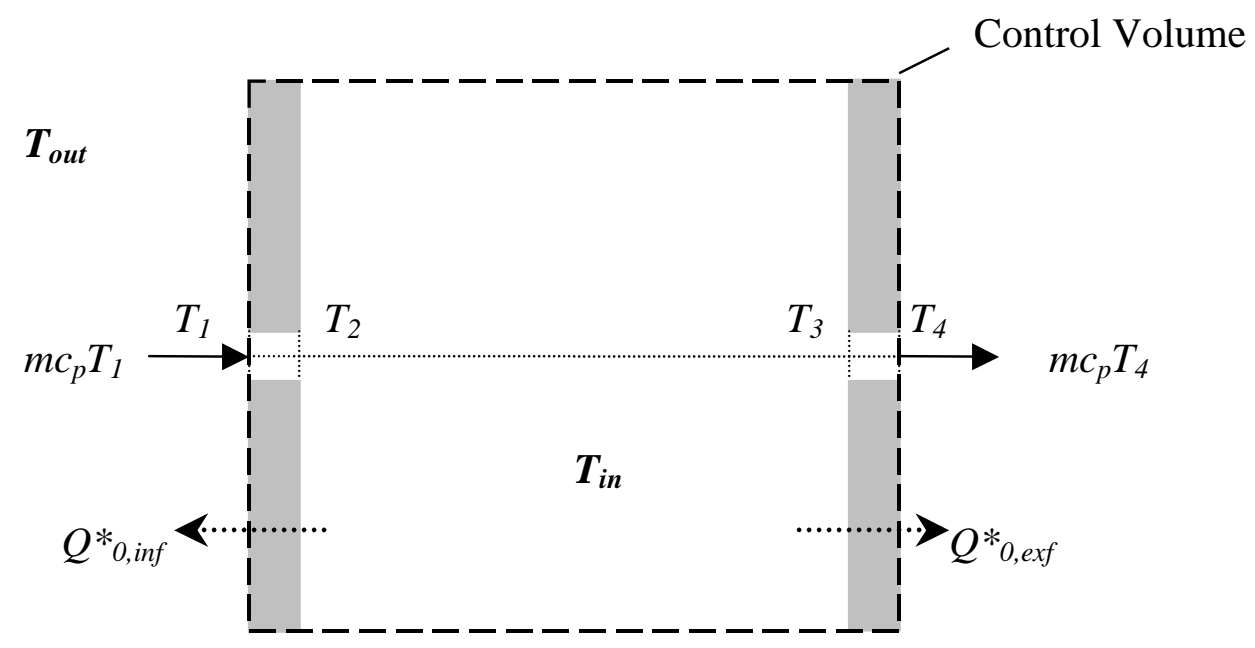

Figure 2: Cross section of a generic building envelope showing the actual conduction and infiltration energy load terms.

To determine the actual conduction heat transfer and its interaction with infiltration heat transfer requires complex analyses and calculations that are not easily carried out. To provide a simple engineering calculation procedure that can be used by practitioners (based on the conventional method given by equation (1)), Sherman et al (2000, 2001) proposed that the conventional method can be corrected by introducing the Infiltration Heat Recovery (IHR) factor. This coefficient undertakes the influence of the infiltrating air on the conduction heat transfer and wall crack temperatures.

$$
Q=Q_{0}+(1-I H R) \times \dot{m} c_{p}\left(T_{\text {in }}-T_{\text {out }}\right)
$$

So that,

$$
I H R=1-\frac{Q-Q_{0}}{\dot{m} c_{p}\left(T_{\text {in }}-T_{\text {out }}\right)}
$$

Note that IHR has been defined such that when the air infiltrating through the walls does not thermally interact with solid components of the wall, IHR reduces to zero, and equation (3) reduces to equation (1). Thus the IHR includes both the effect of temperature changes of the infiltrating and exfiltrating air, and the accompanying changes in conduction that occur. When IHR is non-zero, equation (3) is a convenient way of restating equation (2). The objective of this study was then to take the complex flow and temperature field results from the CFD computations and calculate values of IHR for use in equation (4). 


\subsection{Combining IHR calculations for Infiltrating and Exfiltrating Walls}

The heat exchanges through the infiltrating and exfiltrating walls are calculated separately, and then combined to find the IHR for the whole building by connecting the walls with an indoor space whose other surfaces are adiabatic. The combined effect of the two walls is evaluated by selecting an appropriate control volume. In this study, two of the 4 sides of the control volume coincide with the exterior surfaces of infiltrating and exfiltrating walls. The other two sides are adiabatic (top and bottom). Conduction and convection (i.e. infiltration) heat transfer values are evaluated at the boundaries of the control volume (Figure 3).

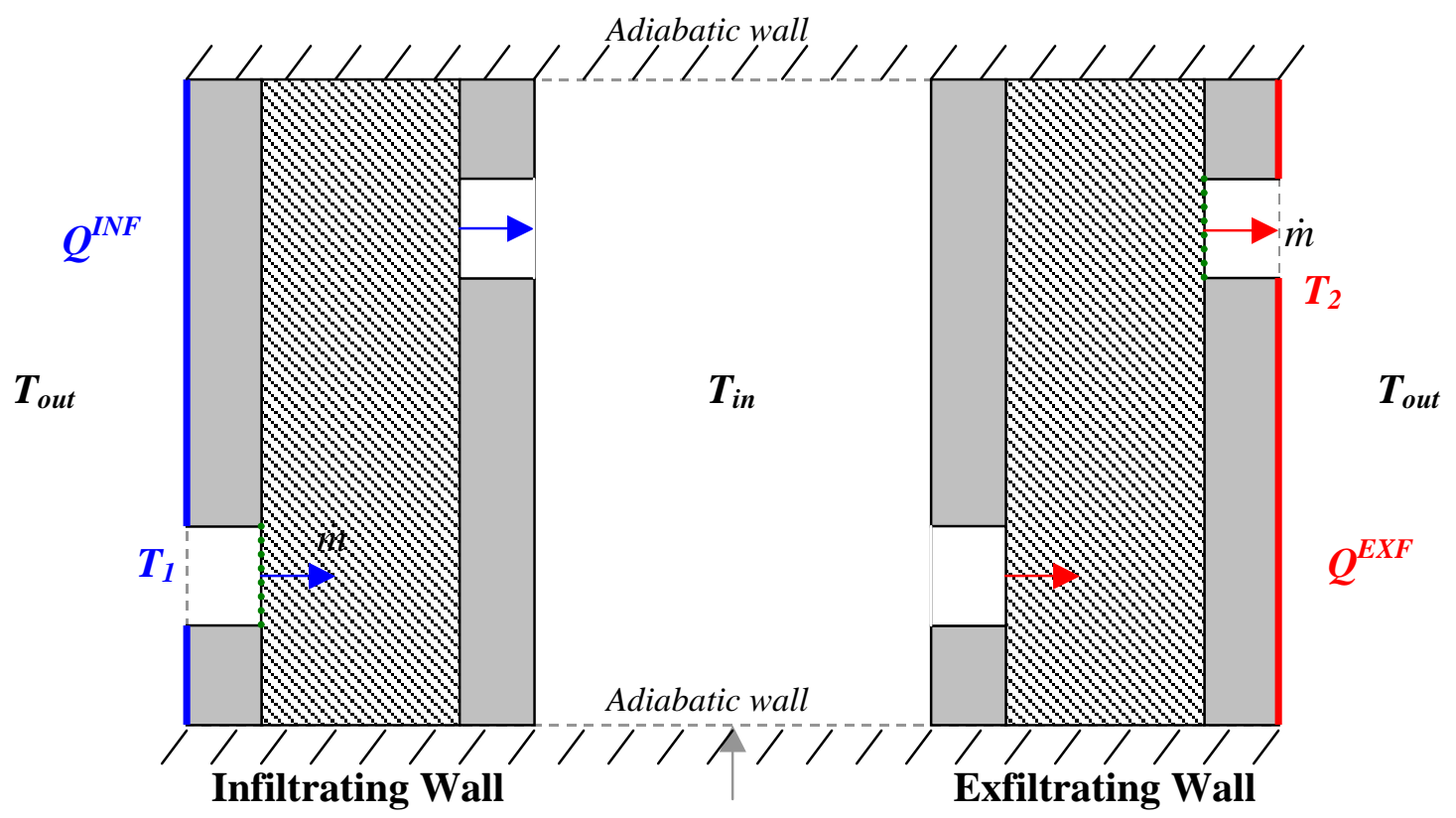

\section{Building Interior}

Figure 3: Heat transfer, air mass flows and temperatures involved in the IHR calculations.

The infiltration heat recovery factors for infiltrating and exfiltrating walls and how they are combined to determine whole building are given by the following equations (based on equation (4)).

$$
\begin{gathered}
I H R_{i}^{I N F}=1-\frac{\dot{m}_{i}^{I N F} \times c_{p} \times\left(T_{\text {in }}-T_{1}\right)+Q_{i}^{I N F}-Q_{0}^{I N F}}{\dot{m}_{i}^{I N F} \times c_{p} \times\left(T_{\text {in }}-T_{\text {out }}\right)} \\
I H R_{j}^{E X F}=0-\frac{\dot{m}_{j}^{E X F} \times c_{p} \times\left(T_{2}-T_{\text {in }}\right)+Q_{j}^{E X F}-Q_{0}^{E X F}}{\dot{m}_{j}^{E X F} \times c_{p} \times\left(T_{\text {in }}-T_{\text {out }}\right)} \\
I H R^{\text {Building }}=\frac{\sum_{i=1}^{n_{\text {INF }}} \dot{m}_{i}^{I N F} \times I H R_{i}^{I N F}}{\sum_{j=1}^{n_{\text {EXF }}} \dot{m}_{j}^{E X F} \times I H R_{j}^{E X F}} \\
\sum_{i=1}^{n_{I N F}} \dot{m}_{i}^{I N F}
\end{gathered}
$$


where $I H R_{i}^{I N F}, I H R_{j}^{E X F}$ and $I H R^{\text {Building }}$ are respectively the infiltration heat recovery factors for infiltrating wall $i$ and exfiltrating wall $j$ and building respectively; $\dot{m}_{i}^{I N F}$ and $\dot{m}_{j}^{E X F}$ are respectively the air mass flux through the infiltrating wall $i$ and exfiltrating wall $j ; c_{p}$ is the air heat capacity, $T_{i n}$ and $T_{\text {out }}$ are the indoor and outdoor temperatures; $T_{1}$ and $T_{2}$ are the infiltrating wall inlet and exfiltrating wall outlet temperatures; $Q_{i}^{I N F}$ and $Q_{j}^{E X F}$ are the heat transfers by conduction through the infiltrating wall $i$ and exfiltrating wall $j$ external faces; $Q_{0}^{I N F}$ and $Q_{0}^{E X F}$ are the infiltrating wall $i$ and exfiltrating wall $j$ heat transfers by conduction without infiltration; and $n_{I N F}$ and $n_{E X F}$ are respectively the number of infiltrating and exfiltrating walls.

The IHR results for the infiltrating and exfiltrating walls and building are presented in terms of the ratio of heat transfer due to air flow to the heat transfer due to conduction: the Peclet number, $P e$, defined by:

$$
P e=\frac{\dot{m} c_{p}}{U A}
$$

where $\dot{m}$ is the air mass flux through the wall $(\mathrm{kg} / \mathrm{s}), c_{p}$ is the air heat capacity $(\mathrm{J} / \mathrm{kgK}), U$ is the wall conductance $\left(\mathrm{W} / \mathrm{m}^{2} \mathrm{~K}\right)$ and $A$ is the wall surface area $\left(\mathrm{m}^{2}\right)$. Usual Peclet number values found in buildings are within the range $0.1-1.0$.

If all walls of a building are identical (same UA value), IHR for a building can be written as a function of Peclet number:

$$
I H R^{\text {Building }}=\frac{\sum_{i=1}^{n_{I N F}} P e_{i}^{I N F} \times I H R_{i}^{I N F}}{\sum_{i=1}^{n_{I N F}} P e_{i}^{I N F}}+\frac{\sum_{j=1}^{n_{E X F}} P e_{j}^{E X F} \times I H R_{j}^{E X F}}{\sum_{j=1}^{n_{E X F}} P e_{j}^{E X F}}
$$

where $I H R_{i}^{I N F}, I H R_{j}^{E X F}$ and $I H R^{\text {Building }}$ are the infiltration heat recovery factors for infiltrating wall $i$ and exfiltrating wall $j$ and building; $P e_{i}^{I N F}$ and $P e_{j}^{E X F}$ are the infiltrating wall $i$ and exfiltrating wall $j$ Peclet numbers; and $n_{I N F}$ and $n_{E X F}$ are the number of infiltrating and exfiltrating walls.

If, however, the walls have different $U A$ values (e.g., owing to different areas, $A$, or a different conductance, $U$ ) then the simplification presented in equation (9) is not possible, and one must use the basic relationship given in equation (7). 


\section{Details of CFD input for the simulated walls}

\subsection{Wall geometry}

Cross sections representing the six wall configurations studied are shown in Figure 4, together with the wall dimensions. The walls are typical of wood frame construction, with a cavity created by having a layer of sheathing on each side of a vertical wooden stud. The dimensions were chosen to be typical of residential exterior wall construction and the leakage locations were chosen to illustrate the extremes of how much of the wall cavity is traversed by the infiltrating and exfiltrating air flows.

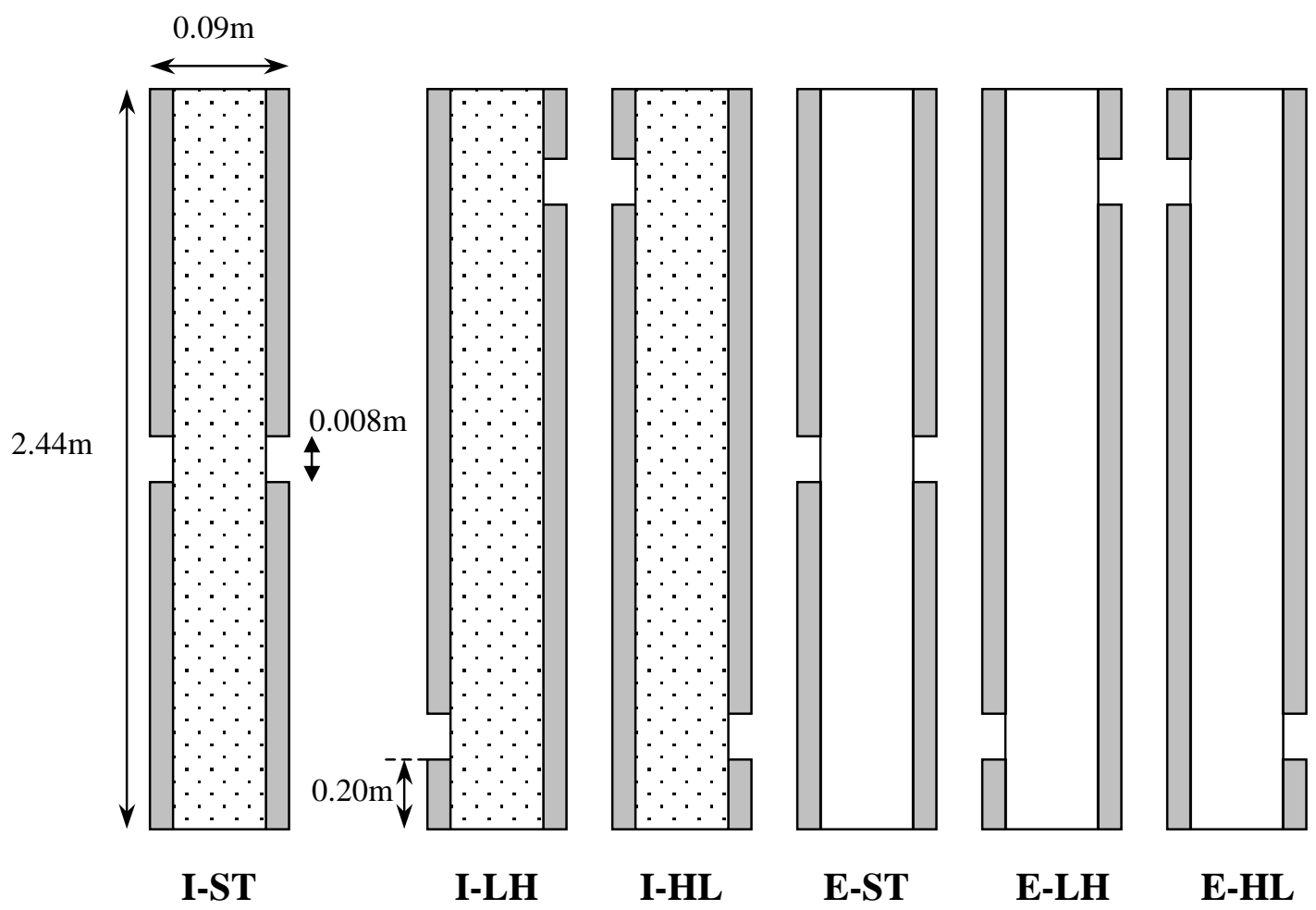

Figure 4: CFD Simulated Wall geometries.

(Notes: I: Insulated wall, E: Empty wall, ST: Straight Through crack, LH: Low/High crack, HL: High/Low crack.)

The three-letter notation below each schematic is a code that succinctly describes the configuration (see text below). Air always enters the wall section from the left crack and exits the wall section from the right crack.

To simplify the discussion of the results, the walls were given code designations corresponding to their insulation and air leak location properties. The first letter in the designation shows whether the wall is Insulated (I) or Empty (E). The position of the inlet and outlet cracks is denoted with the last two letters: Straight Through cracks (ST), Low inlet/High outlet (LH), and High inlet/Low outlet (HL). 


\subsection{Air properties}

\subsubsection{Density}

The ideal gas law is used to calculate the air density:

$$
\rho=\frac{M p_{o}}{R T}
$$

where $\rho$ is the air density $\left(\mathrm{kg} / \mathrm{m}^{3}\right), M$ is the air molar mass $\left(28.9610^{-3} \mathrm{~kg} / \mathrm{mol}\right), p_{o}$ is the atmospheric pressure $(101325 \mathrm{~Pa}), R$ is the ideal gas law constant $(8.314 \mathrm{~J} / \mathrm{molK})$ and $T$ is the air temperature $(\mathrm{K})$.

\subsubsection{Other properties}

All other properties of the air are assumed constant over the temperature range studied in this study. The molecular viscosity is set to $1.8110^{-5} \mathrm{~kg} / \mathrm{ms}$, the specific heat to 1006 $\mathrm{J} / \mathrm{kgK}$ and the conductivity to $0.02637 \mathrm{~W} / \mathrm{mK}$.

\subsection{Sheathing properties}

Sheathing properties are those of plywood: density is $544 \mathrm{~kg} / \mathrm{m}^{3}$, conductivity is $0.13 \mathrm{~W} / \mathrm{mK}$ and specific heat is $1200 \mathrm{~J} / \mathrm{kgK}$.

\subsection{Air flow model}

The Low Reynolds number k- $\varepsilon$ model is employed to calculate the turbulence in the air flows inside the wall cavity and outside the sheathing. This model has the double advantage of (1) allowing the calculation of the boundary layer along walls (the wall region is treated the same way as the interior flow, with a no-slip condition imposed at the boundary; no wall law is applied) and (2) being accurate for Low Reynolds flows (including transitional and laminar flows) characteristic of this problem. According to the STARCD Methodology (1999) manual, the time-averaged basic equations for steady, Low Reynolds number, and incompressible buoyant flows can be written in Cartesiantensor form (equations 11-15). Note that Einstein summation convention is used here and that a comma before an index implies differentiation with respect to the coordinate of the corresponding index. For clarity, we expand the notation in equation (11) into more familiar symbols.

$$
\begin{array}{ll}
\text { Continuity } & \left(\rho U_{i}\right)_{, i}=\sum_{i=1}^{3} \frac{d\left(\rho U_{i}\right)}{d x_{i}}=0 \\
\text { Momentum } & \left(\rho U_{j} U_{i}\right)_{, j}=-P_{, i}-\left(\rho \overline{u_{i} u_{j}}\right)_{, j}+\left(\rho-\rho_{r}\right) g_{i} \\
\text { Energy } & \left(\rho U_{j} T\right)_{, j}=-\left(\rho \overline{u_{j} t^{\prime}}\right)_{, j}
\end{array}
$$


Turbulent Kinetic Energy $\quad\left(\rho U_{i} k\right)_{, i}=\left[\rho\left(\frac{v_{t}}{\sigma_{k}}+v_{l}\right) k_{, i}\right]_{, i}+\rho\left(P_{k}+G_{k}-\varepsilon\right)$

Turbulent Energy Dissipation $\quad\left(\rho U_{i} \varepsilon\right)_{, i}=\left[\rho\left(\frac{v_{t}}{\sigma_{\varepsilon}}+v_{l}\right) \varepsilon_{, i}\right]_{, i}+\rho \frac{\varepsilon}{k}\left(C_{1 \varepsilon} P_{k}+C_{3 \varepsilon} G_{k}-C_{2 \varepsilon} f_{2} \varepsilon\right)$

Equations 16-25 give additional definitions needed to the resolution of the previous system of coupled equations.

$$
\begin{aligned}
& \overline{u_{i} u_{j}}=-v_{t}\left(U_{i, j}+U_{j, i}\right)+\frac{2}{3} k \\
& \overline{u_{j} t^{\prime}}=-\frac{v_{t}}{\sigma_{T}} T_{, j} \\
& \rho=\frac{\rho_{r} T_{r}}{T} \\
& v_{t}=C_{\mu} f_{\mu} \frac{k^{2}}{\varepsilon} \\
& P_{k}=v_{t}\left(U_{i, j}+U_{j, i}\right) U_{i, j} \\
& G_{k}=-v_{t} g_{i} \frac{\rho_{, i}}{\rho \sigma_{T}} \\
& f_{\mu}=\left[1-e^{-0.0198 R_{k}}\right]\left(1+\frac{5.29}{R_{k}}\right) \\
& f_{2}=1-0.3 e^{-R_{t}^{2}} \\
& R_{k}=\frac{y \sqrt{k}}{v_{l}} \\
& R_{t}=\frac{k^{2}}{v_{l} \varepsilon}
\end{aligned}
$$

Where $\rho$ and $\rho_{r}$ are the air density and the reference air density respectively; $U_{i}$ is the mean velocity in the $x_{i}$ direction; $P$ is the static pressure; $T$ and $T_{r}$ are the mean temperature and reference mean temperature; $\overline{u_{i} u_{j}}$ are the Reynolds stresses; $\overline{u_{j} t^{\prime}}$ are the Reynolds heat fluxes; $g_{i}$ is the component $i$ of the gravitation vector; $k$ is the turbulent kinetic energy; $\varepsilon$ is the dissipation of turbulent kinetic energy; $v_{l}$ and $v_{t}$ are the laminar and turbulent viscosity respectively; $\sigma_{k}=1.0, \sigma_{\varepsilon}=1.22$ and $\sigma_{T}=0.9$ are the diffusion coefficients of the turbulent kinetic energy, its dissipation and the temperature respectively; $P_{k}$ is the stress production of turbulent kinetic energy; $G_{k}$ is the buoyancy production of turbulent kinetic energy; $C_{\mu}=0.09, C_{1 \varepsilon}=1.44, C_{2 \varepsilon}=1.92$ and $C_{3 \varepsilon}=0.0$ (or 1.0 if $G_{k}>0$ ) are turbulent model constants; $f_{2}$ is a function used to modified the model constant $C_{2 \varepsilon} ; y$ is the distance to the nearest wall. 
Buchanan and Sherman (2000) showed that in all realistic cases of air infiltration through an insulated wall, the air and insulation temperatures can be assumed to be in local equilibrium. As a consequence, the temperatures of air and insulation are not solved separately in the present calculations and the insulation is treated as a homogeneous porous medium. The air flow calculations are based on the standard Darcy law

The set of equations for the insulated regions is the following:

Continuity $\quad\left(\rho U_{i}\right)_{, i}=0$

Momentum $\quad \frac{\rho v_{l}}{K} U_{i}=-P_{, i}$

Energy $\quad U_{i} T_{, j}=\left(\lambda_{\text {effective }_{, i}} T_{, i}\right.$

where $K$ is the medium's permeability $\left(10^{-8} \mathrm{~m}^{2}\right)$ and $\lambda_{\text {effective }}$ is the effective medium's conductivity based on the solid medium's conductivity $(0.041 \mathrm{~W} / \mathrm{mK})$ and the medium's porosity (0.99).

\footnotetext{
${ }^{1}$ STAR-CD allows the possibility to take the turbulence effect into account on the heat calculations at the interface air/porous medium. Tests showed that this effect is negligible in the present study because of the small size of the wall cracks.
} 


\section{CFD simulations}

CFD simulations were performed with the commercial code STAR-CD, for each of the six wall configurations, for both infiltrating and exfiltrating flows, and for a range of air flows and pressure differences. The building control volume shown in Figure 2 (made up of one infiltrating and one exfiltrating wall) is never simulated in a single CFD run, rather the total heat load on it is determined by combining the results of simulations performed for single walls.

For a heated house in a cold environment, there will be a thermal boundary layer of warm air rising on the outdoor surfaces of the walls. Sherman and Walker (2001) described the possible predominant role of such air boundary layers (along the faces of the wall) in the heat recovery process. This layer, they pointed out, would contribute to the infiltrating air flow entering through the cracks, reducing infiltration heat load. The cool air emerging on the indoor side of the wall will be entrained in the downward moving cool boundary layer, and thus reduce conduction heat loss from wall surface.

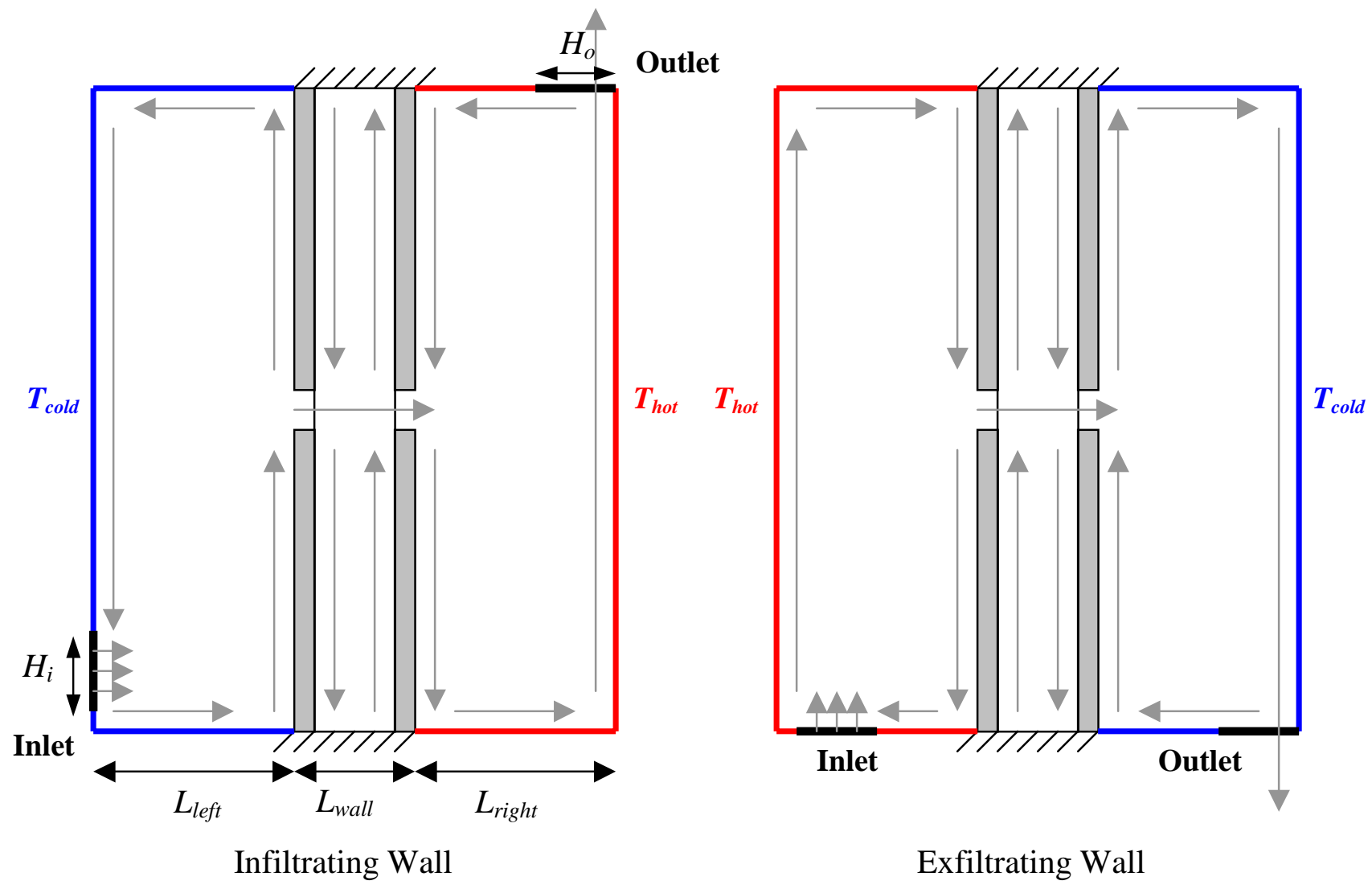

Figure 5: Inlet/outlet locations.

To examine this effect, two different problems are studied. The first case is with no boundary layers in which a single wall is modeled with temperatures imposed directly on its surfaces (no film resistance was applied to represent the boundary layer resistance). The second case adds the external boundary layers to the simulation. Figure 5 illustrates the extension of the computational domain, one on each side of the wall, and shows the 
inlet/outlet location for both the infiltrating and exfiltrating walls and for the computational space boundary. This computation of external flow field is limited to dimensions presented in Table 1 referring to Figure 5. These additional spaces (referred to as cavity in the next sections) are large enough that the boundary layer flows on the wall faces are largely unaffected by the CFD computational space boundaries, and small enough that the increase in CFD computational time is acceptable.

The mass flow of air through the walls is controlled by pressure differentials between the inlet and outlet boundaries of the CFD computation space. A positive pressure is created at the inlet and the outlet pressure is set to zero. Inlet and outlet boundaries are located at the faces of the external wall crack for the no boundary layer (NB) case. For the boundary layer (B) case, the left and right cavity sizes and inlet/outlet sizes and locations have all been optimized by performing successive simulations to fulfill the following goals:

- The main goal is to assure the formation of an unperturbed ascending or descending air boundary layer on the wall. Cavity sizes were increased sufficiently to avoid the air flows at the inlet and the outlet of the computational domain from disturbing the boundary layers formed on the wall surfaces. This was particularly true of the inlet side, since a jet forms from the incoming air and significantly modifies the boundary layer. The inlet size was also increased to reduce the velocity of air entering the external cavity through the inlet, to reduce the disturbance of the wall boundary layers. This increase in inlet size was limited by the formation of two-way flows at the CFD boundary, if the inlet became too large.

- The calculations were found to diverge for small outlet cavities (case of the infiltrating wall) because the pressure cannot be controlled and increases in the outlet cavity as the simulation progresses. As a remedy, cavity size was increased and the outlet location was modified to allow an easier air exit. This eliminated the divergence problem.

Table 1 summarizes the geometries we found to optimize the CFD code performance. The slight difference between the inlet/outlet heights within columns Hi and Ho arises from a small difference in the mesh spacing.

Table 1: External Cavity characteristics for the boundary layer cases.

\begin{tabular}{ccccc}
\hline & $L_{\text {left }}$ & $L_{\text {right }}$ & $H_{i}(m)$ & $H_{o}(m)$ \\
\hline ST & $2 \times L_{\text {wall }}$ & $2 \times L_{\text {wall }}$ & 0.07 & 0.03 \\
LH / HL & $7 \times L_{\text {wall }}$ & $3 \times L_{\text {wall }}$ & 0.076 & 0.05 \\
\hline
\end{tabular}


Table 2 summarizes the cases studied to examine the effects of the boundary layer. Converged computations for a total of 19 configurations are reported including all insulated wall configurations. Only a limited number of simulations for empty wall configurations are reported. We present results for No Boundary layer (NB) cases and a few results for cases including the boundary layers (B). All NB configurations have the same set of pressure differentials across the wall (noted as P1 in Tables 2 and 3). Fewer simulations are reported for the configuration with boundary layer (B) because of limitations imposed by convergence instability and needs for large amounts of simulation time.

Table 2: Summary of configurations simulated to investigate boundary layer effects.

\begin{tabular}{|c|c|c|c|c|c|c|c|}
\cline { 3 - 8 } \multicolumn{2}{c|}{} & \multicolumn{2}{c|}{ ST } & \multicolumn{2}{c|}{ LH } & \multicolumn{2}{c|}{ HL } \\
\cline { 2 - 8 } \multicolumn{2}{c|}{} & INF & EXF & INF & EXF & INF & EXF \\
\hline \multirow{2}{*}{ NB } & I & P1 & P1 & P1 & P1 & P1 & P1 \\
\cline { 2 - 8 } & E & P1 & P1 & P1 & P1 & P1 & P1 \\
\hline \multirow{2}{*}{ B } & I & P2 & P3 & P4 & P4 & P5 & P5 \\
\cline { 2 - 8 } & E & P6 & - & - & - & - & - \\
\hline
\end{tabular}

Notes: NB: No air Boundary layers (temperature imposed directly on wall surfaces), B: air Boundary layers are included in the simulation, I: Insulated wall, E: Empty wall, ST: Straight Through crack, LH: Low/High crack, HL: High/Low crack, INF: INFiltrating wall, EXF: EXFiltrating wall, and Pi $(i=1,2, \ldots 6)$ : Pressure differential imposed across wall cracks (see Table 3 for values).

Table 3: Pressure gradients $(\mathrm{Pa})$ between wall cracks indicated in Table 2.

\begin{tabular}{lccccl}
\hline P1 & P2 & P3 & P4 & P5 & P6 \\
\hline 0.12 & 0.5 & 0.5 & 0.01 & 0.5 & 0.007 \\
0.2 & 1 & 1 & 0.05 & 2 & 0.011 \\
0.5 & 2 & 2 & 0.1 & 5 & 0.031 \\
1 & 10 & 5 & 0.25 & 20 & 0.14 \\
2 & & 10 & 0.5 & & \\
3.5 & & & 2 & & \\
5 & & & 5 & & \\
7.5 & & & 10 & & \\
10 & & & 20 & & \\
20 & & & & & \\
40 & & & & & \\
60 & & & & & \\
\hline
\end{tabular}

\subsection{Determination of the conduction heat flux without infiltration}

The IHR calculation requires the evaluation of the conduction heat flux when there is no infiltration air flow through the wall, and only natural convection air flows induced by the temperature gradient within the studied domain are taken into account. In the absence of any infiltration, heat transfer across the wall, commonly treated as pure conduction, actually includes the effects of convective air currents set up inside the wall cavity. To evaluate this zero infiltration heat flux the inlet/outlet boundaries were replaced by solid walls. As an illustrative example, Figure 6 presents the temperature, velocity and pressure fields near the exterior and in the interior of an Insulated wall configuration, for zero infiltration. 

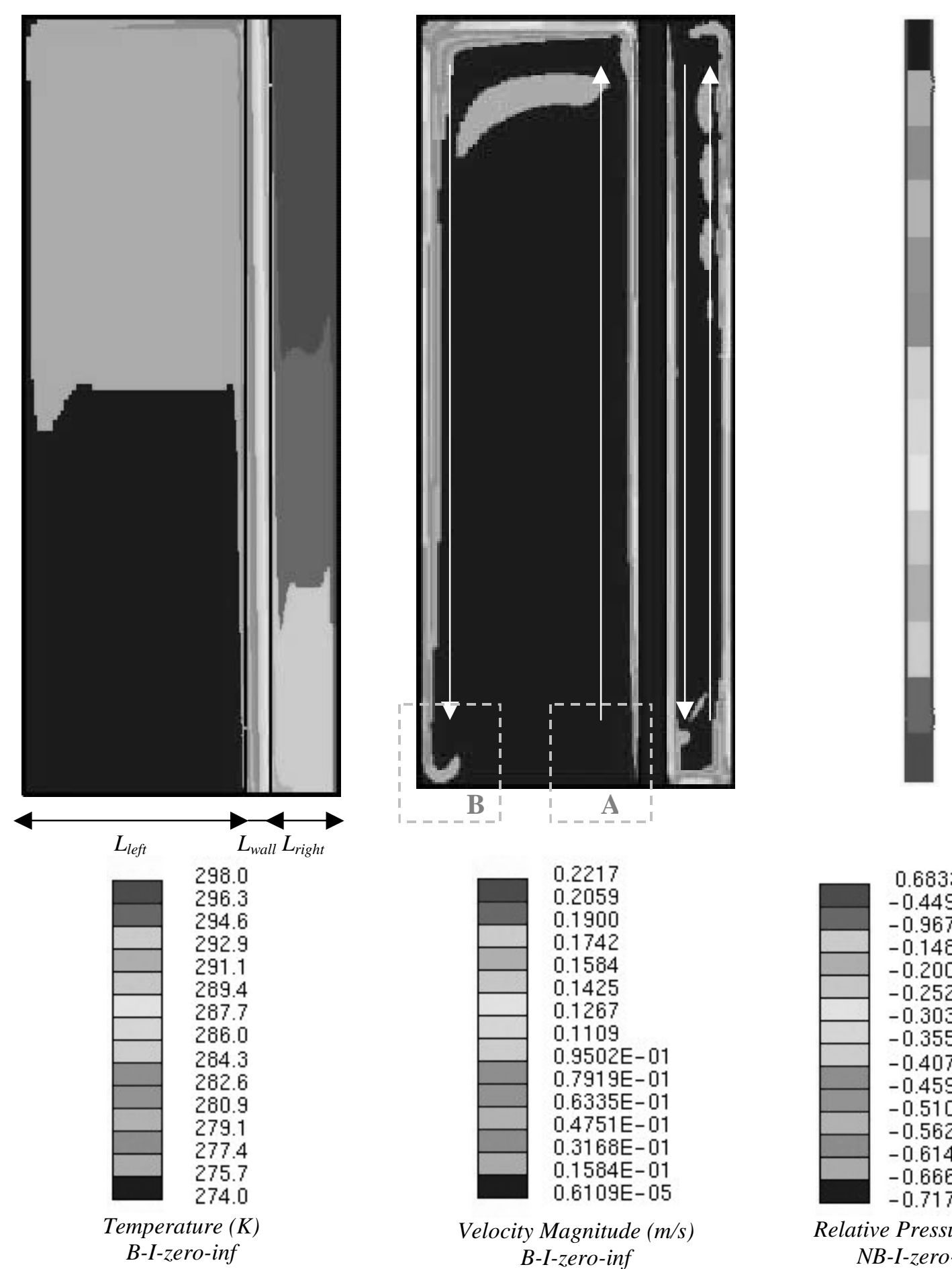

Velocity Magnitude $(\mathrm{m} / \mathrm{s})$ B-I-zero-inf

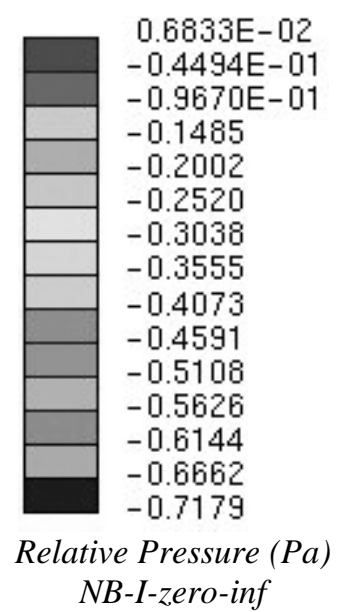

Figure 6: Temperature, velocity and pressure fields for zero infiltration near the exterior and in the interior of an insulated wall. 
The temperature field shows a quasi-uniform temperature in the two cavities with a small gradient due to the change in elevation. The boundary layer effects can be seen close to the solid wall boundaries, isotherms are not exactly vertical contrary to the case with imposed temperature at the wall external faces.

Our goal was that the boundary layers on the vertical boundaries of the computational domain must not interfere with the boundary layers on the exterior of the wall section. The velocity field clearly illustrates that we have met our goal. The external air boundary layers on the wall surfaces are well developed, the boundary layers start with a zero velocity magnitude at the left cavity's bottom right hand corner (zone A in Figure 6) and reach a maximum velocity of $0.2 \mathrm{~m} / \mathrm{s}$. Note that, as we intended, the air flow coming from the opposite walls at the left cavity's bottom left hand corner (zone B in Figure 6) does not perturb the air boundary layer. Similar observations can be made for right cavity's air flow.

The last illustration in Figure 6 shows the vertical pressure gradient in the wall due to buoyancy effects. This pressure gradient between the two cracks for the low/high (LH) and high/low (HL) configurations exists even if there's no imposed air flow. When examining IHR as a function of pressure difference across the leaks, a pressure shift equal to this "pure conduction" pressure gradient will be subtracted so the pressure difference is just the forced convection pressure difference. 


\subsection{Calculation reliability}

\subsubsection{Simulation convergence}

The boundary layer (B) and non-boundary layer (NB) configurations were studied as two different problems. It was easier to achieve a good and quick convergence for the configuration without boundary layers. It was possible to obtain residuals for all variables (velocity, pressure, turbulent kinetic energy, turbulent energy dissipation and temperature) lower than $10^{-6}$ in 10-20 minutes using one processor on a Silicon Graphics Origin 2000 server (called LORAX in the rest of this text), with 2 processors, $2000 \mathrm{MB}$ of RAM and a $100 \mathrm{~GB}$ external hard drive. This convergence level was not obtained for one configuration and one imposed pressure gradient: the NB-E-ST-INF at 1.0 Pa. This failure to converge is linked to a physical change of the air flow at or near this particular pressure gradient. This is most likely a case where no steady state situation exists or there are multiple stable solutions to the air flow and heat transfer.

Figure 7 illustrates the air flow structures and turbulence intensity inside an empty wall with a straight-through crack as a function of pressure gradient spanning this poor convergence case. This figure illustrates a central portion of the wall (rather than its complete height) in order to make it easier to see the air flow structure. Note that color scale is not the same for every case, color is only used to identify the turbulent structures. In the zero infiltration case $(0.0 \mathrm{~Pa})$, air gets cold near the left face and descends while it gets hot near the right face and rises. As a consequence a clockwise loop is created. For low-pressure gradients (below 1.0 Pa), the loop is cut into two clockwise loops and the turbulence in the region near the crack is small compared to that in the loops. For pressure gradients closer to 1.0, calculation convergence cannot be achieved. Tests show that this instability occurs for pressure gradients between 0.8 and $1.2 \mathrm{~Pa}$. We believe that the cause of this unstable air flow lies in the appearance of two new loops near to the crack. For pressure gradient of 2.0, the air flow through the cracks is strong and cuts the thermal flow within the wall cavity in two regions. These two loops can also be seen at higher pressure gradients.

The configuration with boundary layers is much more difficult to simulate because accounting for the external air boundary layer makes the problem much more complex. Numerous geometrical adjustments (e.g. enlarging the left cavity or changing inlet/outlet sizes and locations) and calculation methods (e.g. using zero infiltration converged solution or previous converged solution for initialization) were necessary to obtain results. It's difficult to achieve a good convergence everywhere in the domain, particularly in the center of the cavities and at the inlet boundary region. The method to assess a good convergence for this configuration was to look for and confirm well developed boundary layers in the near wall regions. The simulations were also time consuming and each took a minimum of 14 hours using one processor on LORAX. Good convergence was harder to achieve with the lowest pressure gradients, and reliability of the results for low pressure gradients is worse than for higher pressure gradients. This is because it's difficult to model very small air flows (low velocities and low overall kinetic energy), when much of the flow field is close to resolution limit of the CFD code. 


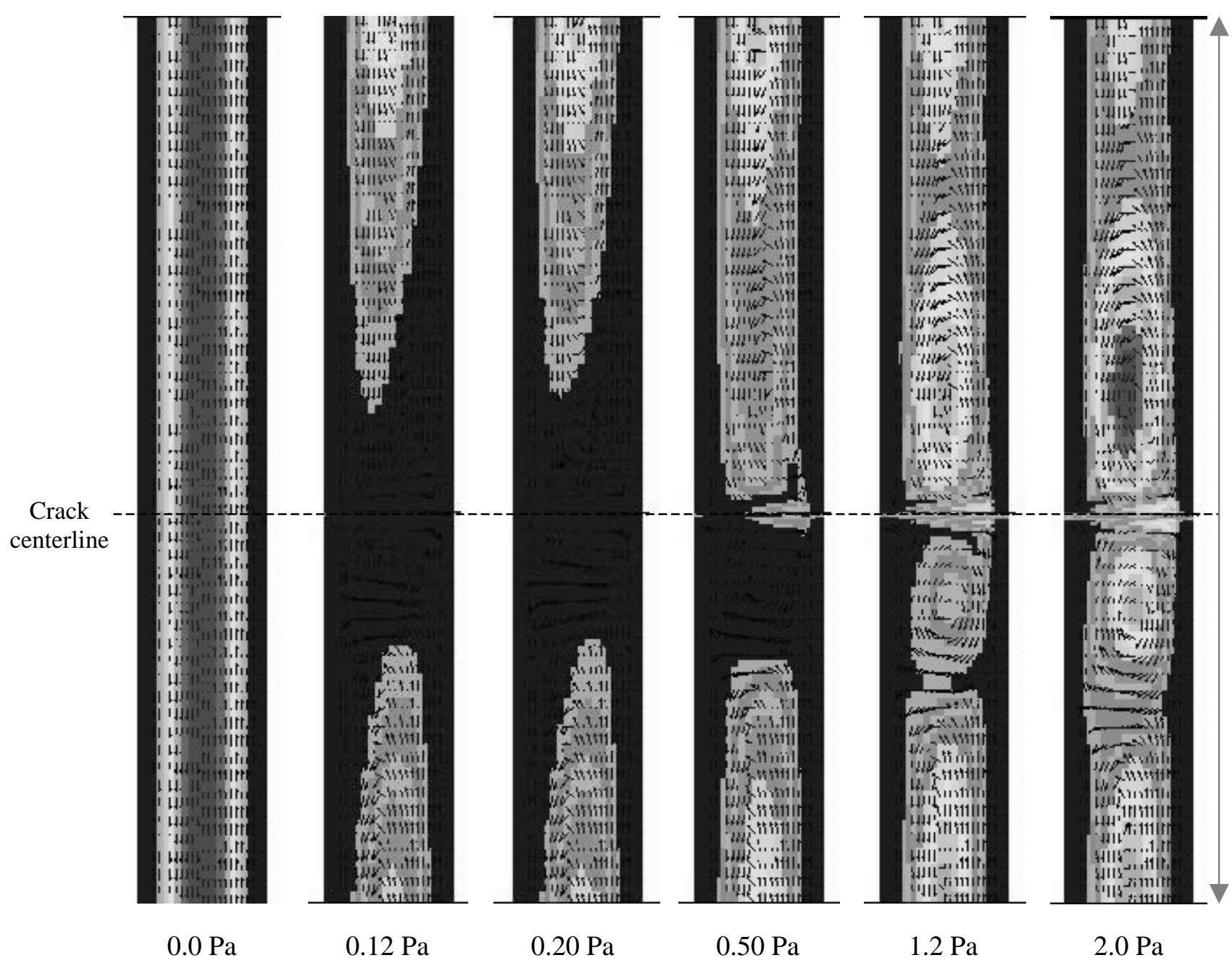

$0.6 \mathrm{~m}$

Figure 7: Turbulent structures in the wall cavity (NB-E-ST-INF). For clarity, only the central section of the wall is shown enlarged.

\subsubsection{IHR Error estimates}

This section is dedicated to the evaluation of uncertainties in predicted IHR values. Calculations are based on the calculation procedure used to calculate the IHR (equations (5) and (6)) and on the uncertainties in the CFD modeling. Results show that IHR values can be obtained with reasonable accuracy (lower than 20\%) only for Peclet numbers higher than 0.1 .

Error propagation is used to combine random errors of CFD parameters to get an estimate for the total error. It is used in situations where one doesn't have the luxury or ability to measure the same thing several times and thereby estimate the random error on one's final result directly. 
Error propagation can also be used to combine several independent sources of random error on the same measurement. The random error calculation principles are the following. Let $f, g$ and $h$ be three functions. Function $f$ is obtained from functions $g$ and $h$. Then the random error on $f, \Delta f$, is a function of the random errors on $g, \Delta g$, and $h, \Delta h$, depending on the functional dependence of $f$ on $g$ and $h$ as follows:

$$
\begin{array}{lll}
f=g+h & \Rightarrow & (\Delta f)^{2}=(\Delta g)^{2}+(\Delta h)^{2} \\
f=\frac{g}{h} & \Rightarrow & \left(\frac{\Delta f}{f}\right)^{2}=\left(\frac{\Delta g}{g}\right)^{2}+\left(\frac{\Delta h}{h}\right)^{2} \\
f=a g & \Rightarrow & \Delta f=a \Delta g
\end{array}
$$

Applying these rules to equations (5) and (6), the random errors for the infiltrating and exfiltrating walls are given by:

$$
\begin{aligned}
\left(\Delta I H R^{I N F}\right)^{2}=\left(\frac{\Delta T_{1}}{T_{\text {in }}-T_{\text {out }}}\right)^{2} & +\left(\frac{Q^{I N F}}{\dot{m}}\right)^{2}\left(\left(\frac{\Delta Q^{I N F}}{Q^{I N F}}\right)^{2}+\left(\frac{\Delta \dot{m}}{\dot{m}}\right)^{2}\right) \\
& +\left(\frac{Q_{0}}{\dot{m}}\right)^{2}\left(\left(\frac{\Delta Q_{0}}{Q_{0}}\right)^{2}+\left(\frac{\Delta \dot{m}}{\dot{m}}\right)^{2}\right)(32) \\
\left(\Delta I H R^{E X F}\right)^{2}=\left(\frac{\Delta T_{2}}{T_{\text {in }}-T_{\text {out }}}\right)^{2} & +\left(\frac{Q^{E X F}}{\dot{m}}\right)^{2}\left(\left(\frac{\Delta Q^{E X F}}{Q^{E X F}}\right)^{2}+\left(\frac{\Delta \dot{m}}{\dot{m}}\right)^{2}\right) \\
& +\left(\frac{Q_{0}}{\dot{m}}\right)^{2}\left(\left(\frac{\Delta Q_{0}}{Q_{0}}\right)^{2}+\left(\frac{\Delta \dot{m}}{\dot{m}}\right)^{2}\right)
\end{aligned}
$$

The main difficulty lies in the determination of the random error for the variables evaluated by CFD calculations. Precision of the results depends on the specified variable. Fractional uncertainties, $\left(\frac{\Delta f}{f}\right)$, are lower than:

- $\quad 10^{-5}$ for temperature and conduction and

- $10^{-6}$ for mass flow.

Accuracy of the CFD results depends on a host of other feature of the simulation, such as adequacy of the turbulence model, adequacy of grid geometry and so on. Looking only at the CFD convergence error can not capture these errors from model misfits. Experiment comparisons are necessary for good estimation of errors from model misfits. Since we do not have an estimate of error from model misfit, we use the CFD convergence error as the minimum estimate of error. Actual errors are probably several times larger. 
Reasonable absolute uncertainty ( $\Delta f$ ) estimation based on typical results is given below:

$$
\begin{array}{ll}
- & \Delta T=5 \times 10^{-3} \mathrm{~K} \\
- & \Delta Q=\Delta Q_{0}=5 \times 10^{-6} \mathrm{~W} \\
- & \Delta \dot{m}=5 \times 10^{-14} \mathrm{~kg} / \mathrm{s}
\end{array}
$$

Figure 8 presents the IHR random error for infiltrating configurations as a function of Peclet number.

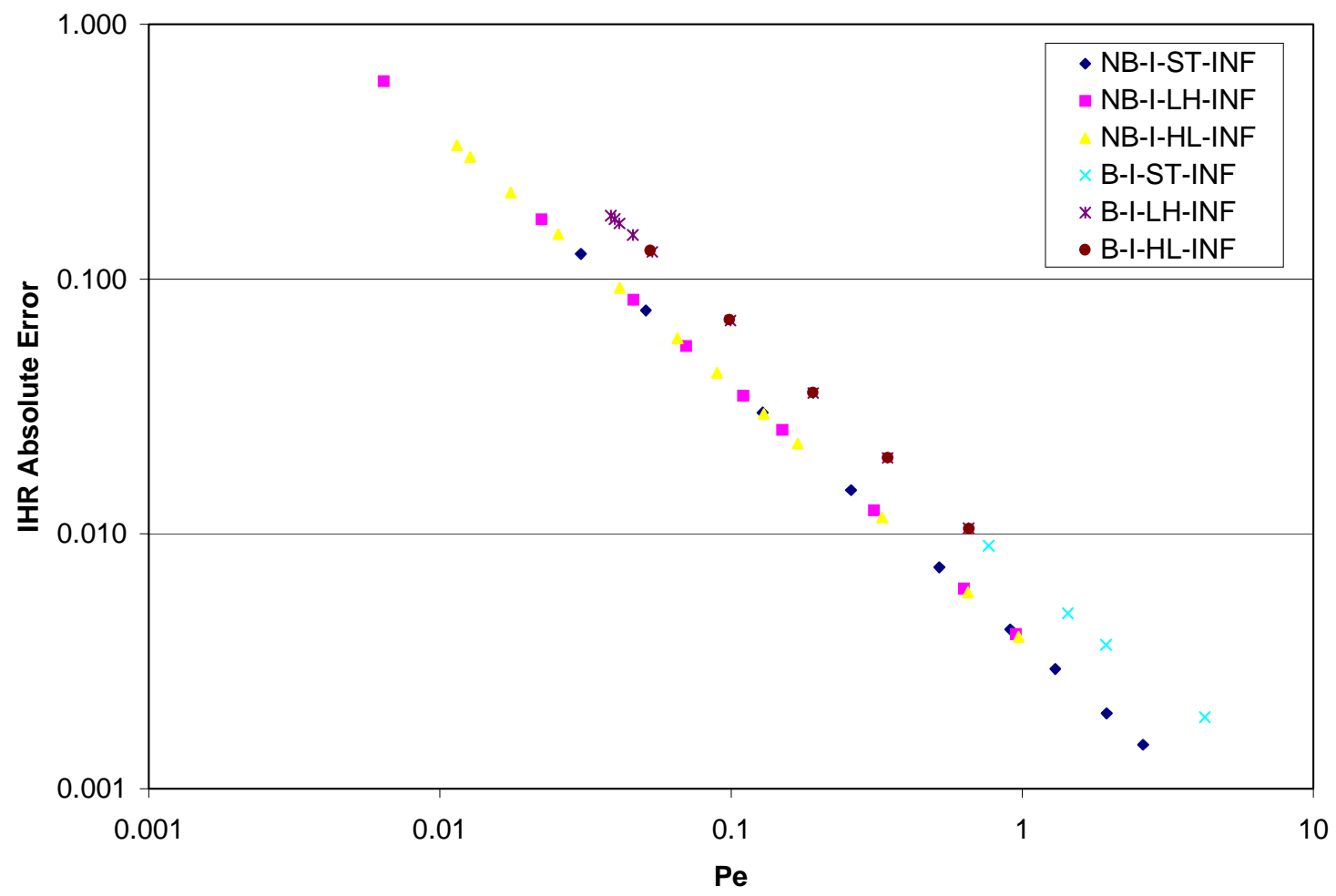

Figure 8: Absolute random error as a function of Peclet number.

(Notes: NB: No air Boundary layers (temperature imposed directly on wall surfaces), B: air Boundary layers are included in the simulation, I: Insulated wall, ST: Straight Through

crack, LH: Low/High crack, HL: High/Low crack, INF: INFiltrating wall.)

The separation in Figure 8 between the curves for no boundary (NB) and boundary (B) cases is due to the change in wall conduction value. Because of the boundary layers, the wall conductance value in case B is lower than the no boundary (NB) one. Note that even if the random error decreases rapidly with an increase of the Peclet number, uncertainty is very high for Peclet number lower than 0.1. As an example, IHR uncertainty is $50 \%$ for Peclet number 0.01 and decreases to $20 \%$ for Peclet number 0.1 . Thus IHR values can be obtained with reasonable accuracy $(20 \%)$ only for Peclet numbers higher than 0.1 . 


\section{Results}

\subsection{Insulated Wall}

\subsubsection{Straight Through crack}

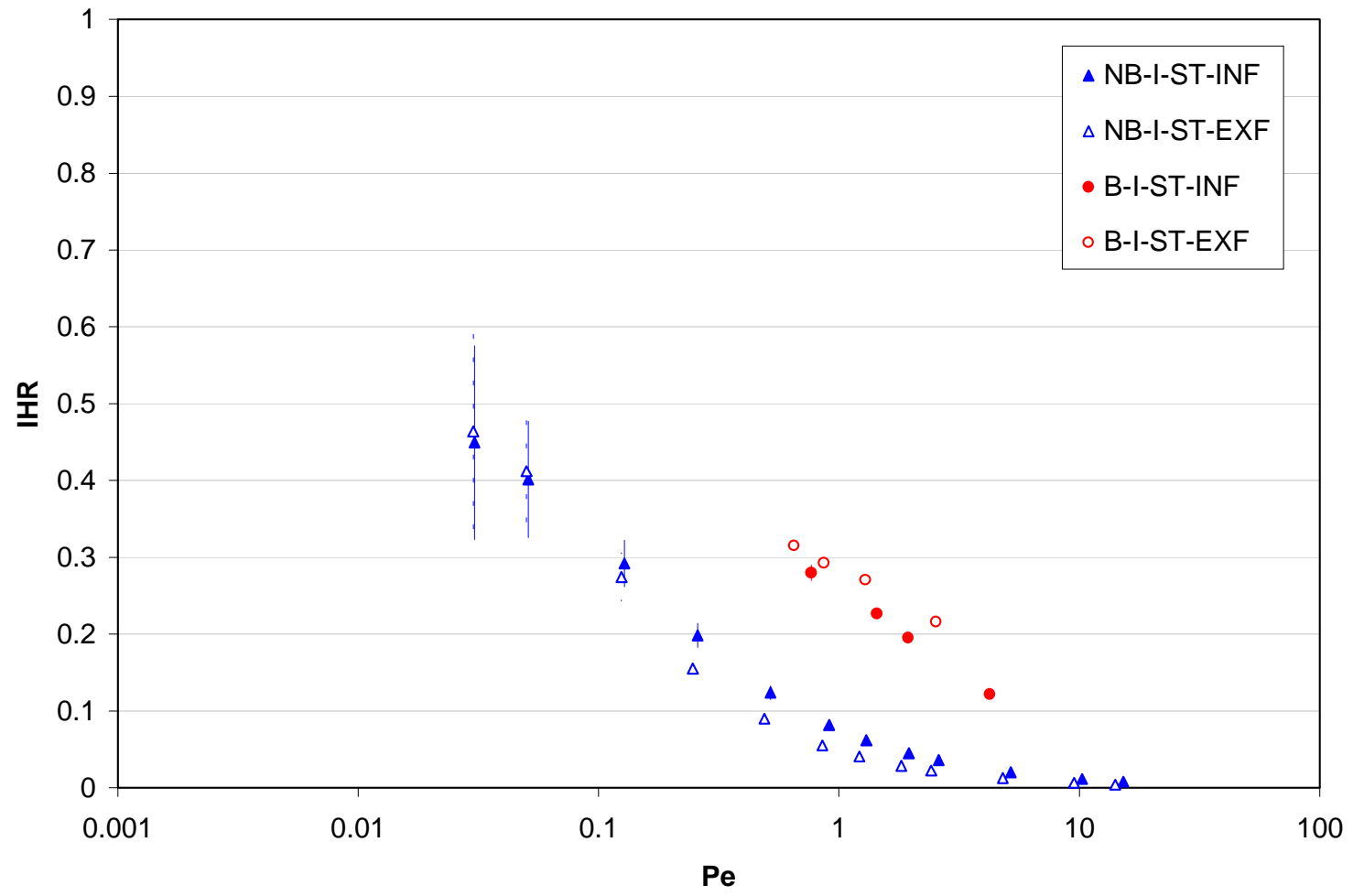

Figure 9: IHR results for Insulated walls, with Straight Through leaks (I-ST).

(Notes: NB: No air Boundary layers (temperature imposed directly on wall surfaces), B: air Boundary layers are included in the simulation, I: Insulated wall, ST: Straight Through crack, INF: INFiltrating wall, EXF: EXFiltrating wall.)

Figure 9 shows all the CFD results for the straight through crack cases. The vertical lines indicate the uncertainty limits discussed earlier. Points with no visible lines mean that the uncertainty limits are smaller than the size of the points used in the figure. IHR values increase towards 0.5 as Peclet number decreases. This is consistent with the limiting value of 0.5 for low-Peclet numbers as suggested from the analytical approach of Sherman and Walker (2001). At a given Peclet number, the IHR values are higher for the infiltrating wall than for the exfiltrating wall. This tendency is observed for all walls with no boundary layers (NB).

Results for the walls with boundary layers (referred to in the rest of the text as the boundary layers (B) case) follow the same trends. IHR values are larger by about 0.2 than for the no boundary layers case (NB). This shows that the effect of the external air boundary layer on the IHR is significant. For the infiltrating wall (given the inside warm and the outside cold), the added heat recovery is the result of the air entering the wall from the outdoor warm boundary layer, which is at a higher temperature than the cold ambient air. On the room side, the cooler air entering through the leak enters the indoor 
cool boundary layer and acts to reduce conduction losses through the wall. The same effects also occur for the exfiltrating wall.

The CFD code encountered a convergence problem for the B-I-ST-EXF configuration at high-Peclet number (for Peclet number close to 3). Additional simulations were conducted to address this problem: calculations were performed using more iterations, convergence criteria were tightened and pressure boundary conditions were replaced by velocity boundary conditions to avoid two-way flows at the domain boundaries. None of these modifications were able to address the convergence problem for this case at high Peclet numbers.

\subsubsection{Low/High and High/Low cracks}

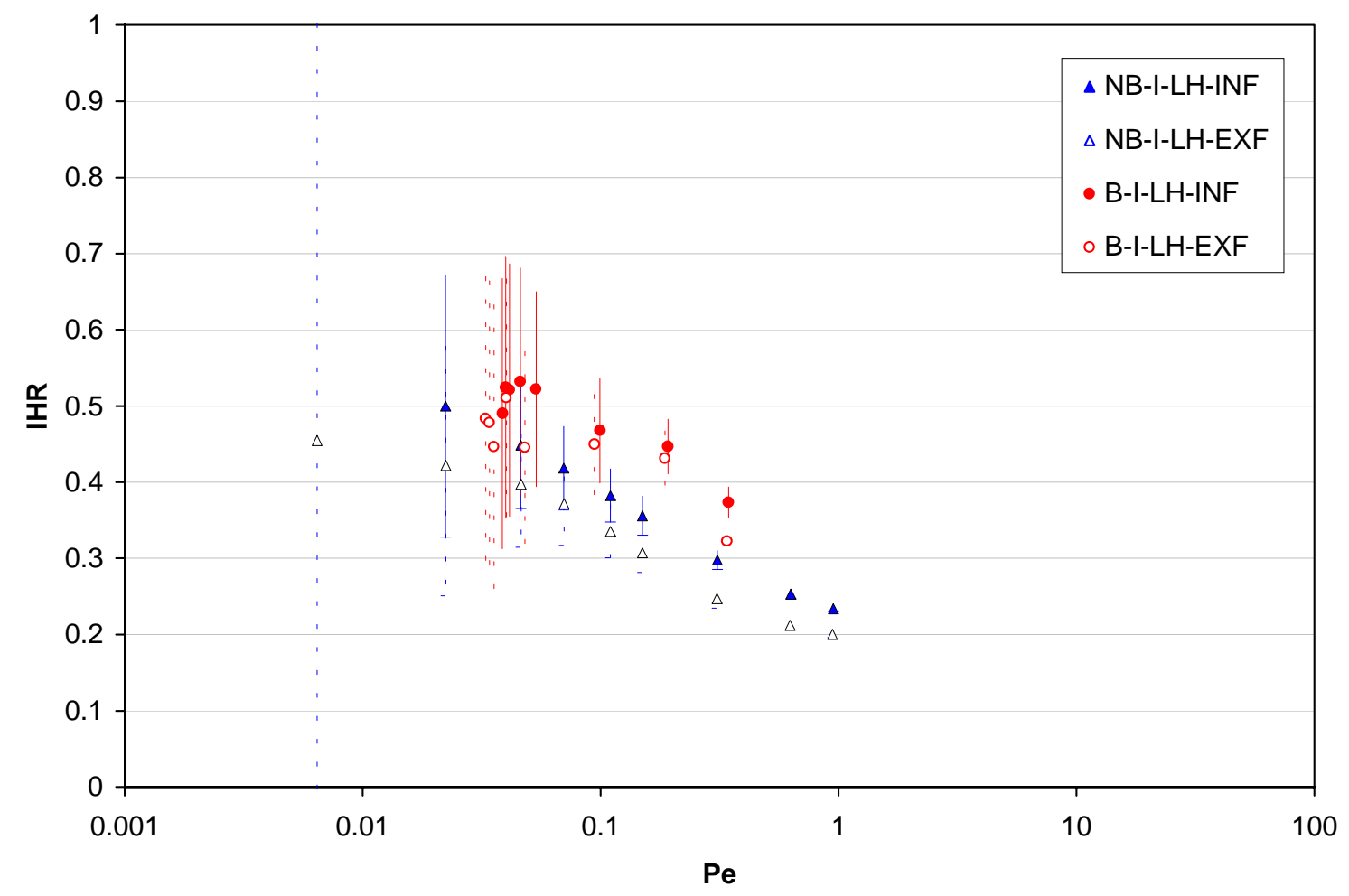

Figure 10: IHR results for Insulated walls and air flowing from Low to High leaks (I-LH). (Notes: NB: No air Boundary layers (temperature imposed directly on wall surfaces), B: air Boundary layers are included in the simulation, I: Insulated wall, LH: Low/High crack, INF: INFiltrating wall, EXF: EXFiltrating wall.)

The results for these geometries are shown in Figure 10, together with vertical lines showing the uncertainty estimates. As for the straight through case, IHR values for infiltrating wall are higher than those for exfiltrating wall and the difference remains about the same for the entire Peclet number range. Also, as in the previous case, results are consistent with a limit value of 0.5 for low-Peclet number. Predicted errors are higher than for the straight through (ST) configuration. The high flow resistance of the longer air flow path lengths for these cases leads to very low infiltrated air mass flux. 
Results for the boundary case (B) follow the same trend, with IHR values about 0.1 higher than for the no boundary case (NB). Values have more scatter for low-Peclet number but are close to 0.5. Convergence problems appeared for the boundary case (B) at the highest Peclet numbers as they did for the straight through configuration (ST).

Figure 11 presents the results for NB-HL configuration that are very similar to the NB-LH configuration. IHR results for NB-I-HL-INF and B-I-HL configurations are greater than the theoretical maximum 0.5 for low-Peclet numbers. At these low-Peclet number the estimated minimum errors encompass a range including the theoretical 0.5 limit for IHR. However, the data show distinct trends to IHR higher than 0.5 rather than having more scatter indicative of randomness. These trends therefore indicate some sort of additional systematic uncertainty in the CFD results.

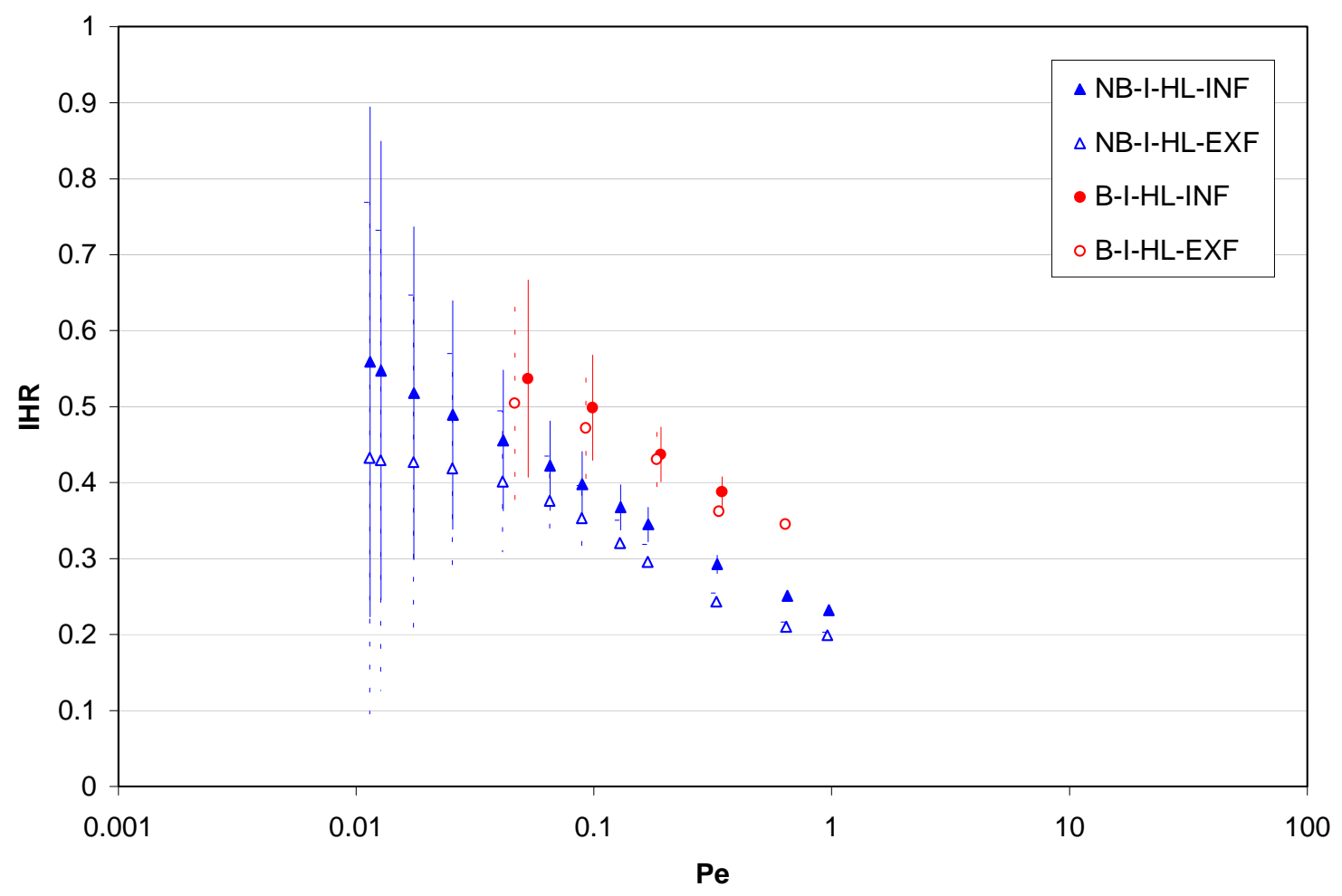

Figure 11: IHR results for Insulated walls and air flowing from High to Low leaks (I-HL). (Notes: NB: No air Boundary layers (temperature imposed directly on wall surfaces), B: air Boundary layers are included in the simulation, I: Insulated wall, HL: High/Low crack, INF: INFiltrating wall, EXF: EXFiltrating wall.)

\subsection{Empty Walls}

IHR results for an empty wall presented below are for no boundary cases (NB), except for the straight through configuration (ST) where approximated values based on not-fully-converged computations are shown for boundary case (B). 


\subsubsection{Straight Through crack}

Because of the low flow resistance of straight through cracks, the application of typical infiltration pressure differences leads to high Peclet numbers and correspondingly low IHR. Figure 12 illustrates that IHR values for infiltrating wall are higher than for exfiltrating wall.

Converged solutions could not be found for all cases of NB configuration. At lower Peclet numbers for the infiltrating wall we found that two-way flows appeared at the cracks and IHR values for Peclet number lower than 0.5 could not be obtained due to instability in the CFD calculations. Good convergence was reached for the exfiltrating case for the full range of Peclet number values.

Simulations for the boundary layer (B) case also showed two-way flows at the cracks for Peclet number close to 0.5 . Because of these instabilities and limited time, only three IHR results are available. Note that the presence of the boundary layers has an effect on IHR similar to that for the insulated configuration: about a 0.2 increase in IHR value with the external air boundary layer (B) taken into account, compared to the NB cases.

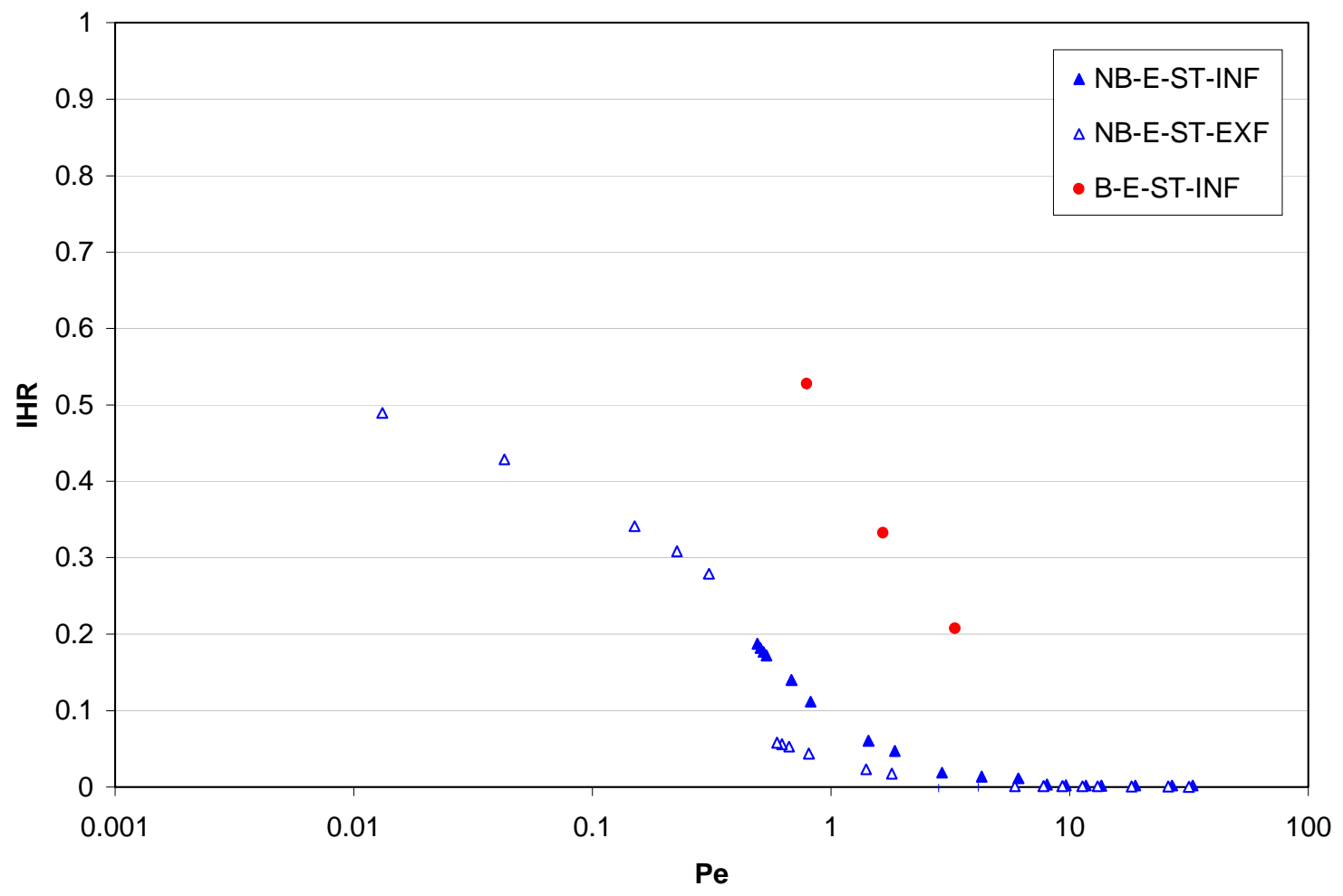

Figure 12: IHR results for Empty walls for Straight Through leaks (E-ST).

(Notes: NB: No air Boundary layers (temperature imposed directly on wall surfaces), B: air Boundary layers are included in the simulation, E: Empty wall, ST: Straight Through crack, INF: INFiltrating wall, EXF: EXFiltrating wall.) 


\subsubsection{Low/High and High/Low Cracks}

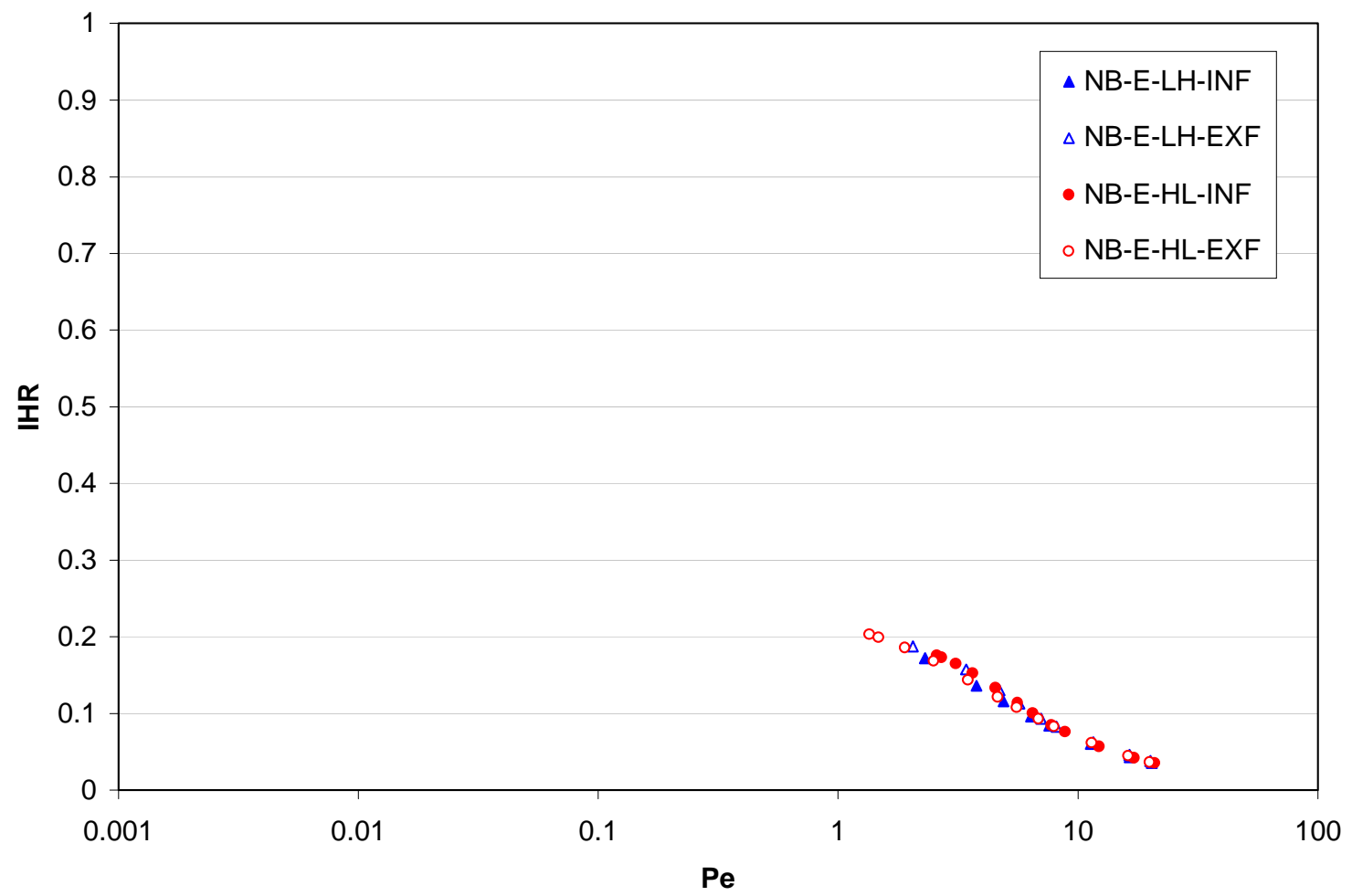

Figure 13: IHR results for Empty walls for Low-High and High-Low leaks (E-LH \& E-HL). (Notes: NB: No air Boundary layers (temperature imposed directly on wall surfaces), E: Empty wall, LH: Low/High crack, HL: High/Low crack, INF: INFiltrating wall, EXF: EXFiltrating wall.)

Results for empty walls with Peclet number higher than one, present the same trend for both infiltrating and exfiltrating walls and low/high (LH) and high/low (HL) configurations. As expected, the IHR values are higher than those for straight through configuration (ST) - like those for insulated walls. Also, as expected, overall the IHR values for empty walls are slightly lower than corresponding values for insulated walls. 


\subsection{Conclusion and future developments}

Buchanan and Sherman (2000) concluded that the interior details of the wall encountered in the leakage path (i.e. insulated or empty walls) do not have a great effect on the heat recovery if external boundary layers are ignored. The overall crack geometry (i.e. straight through (ST) or low/high (LH) and high/low (HL) configurations) is the key factor. This study confirms their observations and shows the important effect of the external boundary layers on conduction and infiltration heat loads.

Comparison between no boundary (NB) and boundary (B) configurations shows that the effect of the boundary layers on the heat recovery is very important (it typically increases IHR by 0.2). Note that this effect is higher for the ST configuration than for the low/high (LH) and high/low (HL) configurations. In the low/high (LH) and high/low (HL) configurations, the air temperature at the outlet crack is close to the air boundary layer temperature because the transit time of air inside the wall is long. As a consequence, the leaking air does not substantially change the boundary layer temperature. Even if the effect of the intermixing between the external boundary layers and the infiltrating air is just located on the half of the wall height for the straight through configuration (ST), the temperature difference is higher and thus its effect becomes predominant. The Simplified Infiltration Heat Recovery Model (proposed by Sherman and Walker 2001) should be modified to take this effect into account at least for the straight through configuration (ST).

This study covered a large range of important factors, nevertheless additional work is required to develop a better understanding of IHR:

- Some CFD results have to be recalculated. This includes all results that do not follow the trends observed in other data: i.e., the last points of the B-I-LH and B-I-HL configurations. Convergence criteria may have to be tightened for these cases or using imposed mass flux as boundary conditions instead of imposed pressure may correct this problem.

- The present database has to be completed. Additional simulations are necessary for the B-I-LH/HL configuration (higher Peclet number) and the Empty wall configuration (lower Peclet number) to fill the Peclet number range. B-E configuration remains to be studied.

- External air boundary layer effect can be examined for the straight through configuration (ST) by preventing the spread of infiltrated air inside the entire wall. This can be accomplished by lining the crack and the wall-passage with adiabatic surfaces to connect the crack.

- Horizontal flow configurations (i.e. floor and ceiling) have to be studied.

These additional simulations can be performed with the existing STAR-CD geometry and models. There is no need to create new meshes (except for the horizontal configurations). 


\section{Illustrative Applications}

This section describes how to apply the previous results to evaluate the Infiltration Heat Recovery effect for a particular building (a detailed example is given in Appendix 1). We illustrate this with two cases, where infiltration is caused by external wind incident normal to one wall of a square building. The first case is the simplest way of representing air leaks in one building and has only one infiltrating and one exfiltrating wall. The second case is a little more realistic, with participation from all four walls. However, no air leaks are considered through the roof and floor for both cases. The studied building has a square footprint, and all four vertical walls have the same area and crack configurations. In both cases, wind pressure is assumed uniform over any given wall.

\subsection{Combining One Infiltrating and One Exfiltrating Wall to Obtain a whole building IRH value}

Figure 14 presents a plan view of the first case. Outdoor air enters the building through the upwind wall (wall 1) and leaves it by the opposite wall (wall 2). Infiltrating and exfiltrating air mass fluxes are equal.

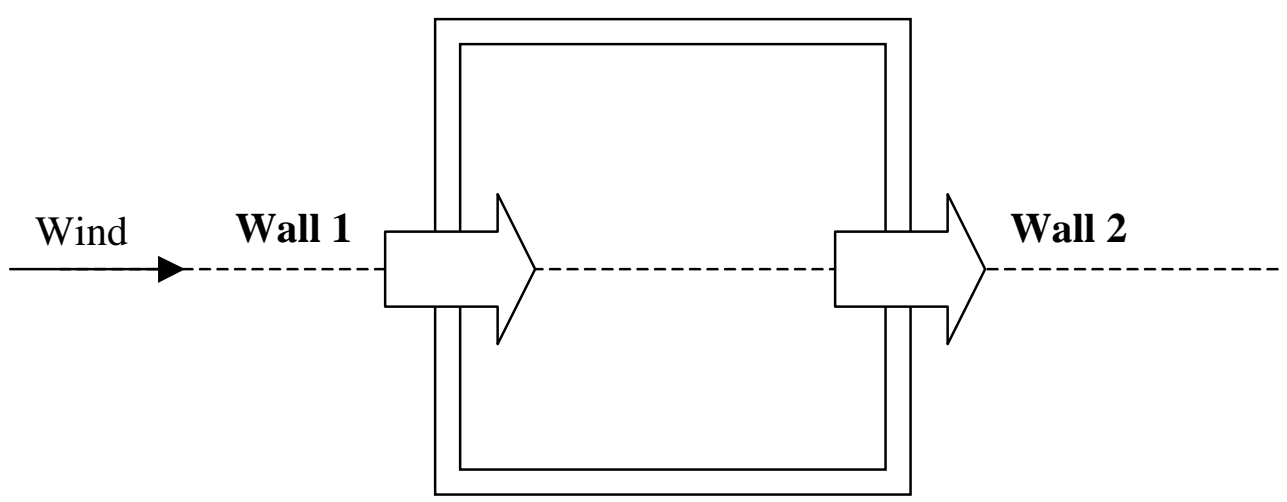

Figure 14: Case 1 - plan view.

For one infiltrating and one exfiltrating walls equation (9) leads to:

$$
I H R^{\text {Building }}=I H R_{1}^{I N F}+I H R_{2}^{E X F}
$$

where $I H R_{1}^{I N F}, I H R_{2}^{E X F}$ and $I H R^{\text {Building }}$ are the infiltration heat recovery factors for infiltrating and exfiltrating walls and building.

Since the two walls are otherwise identical (area, insulation properties and insulation thickness), the infiltrating and exfiltrating flow will have identical Peclet numbers. IHR for a building at a fixed Peclet number is then the direct summation of IHR for infiltrating and exfiltrating walls at the same Peclet number. 
The IHR values for a whole building are calculated by combining the heat flows for an infiltrating and exfiltrating walls.

The following section summarizes trends in IHR values as functions of Peclet number, infiltrated air mass flux, and pressure difference across the wall. Only reliable IHR values are presented, others, which will need further investigation, are excluded from plots for clarity.

\subsubsection{IHR as a function of Peclet number}

Buchanan and Sherman (2001) showed that Peclet number as defined by equation (7) is a useful independent variable to present IHR results because it collapses the data showing the universal trends. Whatever the wall type (insulated or empty), the results show that low/high (LH) and high/low (HL) values are similar - which indicates that the problem is symmetric. For Peclet number higher than 0.1, IHR values are higher for LH/HL configuration than for straight through (ST) one. IHR values for straight through (ST) configuration decrease faster than those for low/high (LH) and high/low (HL) configuration with increasing Peclet numbers.

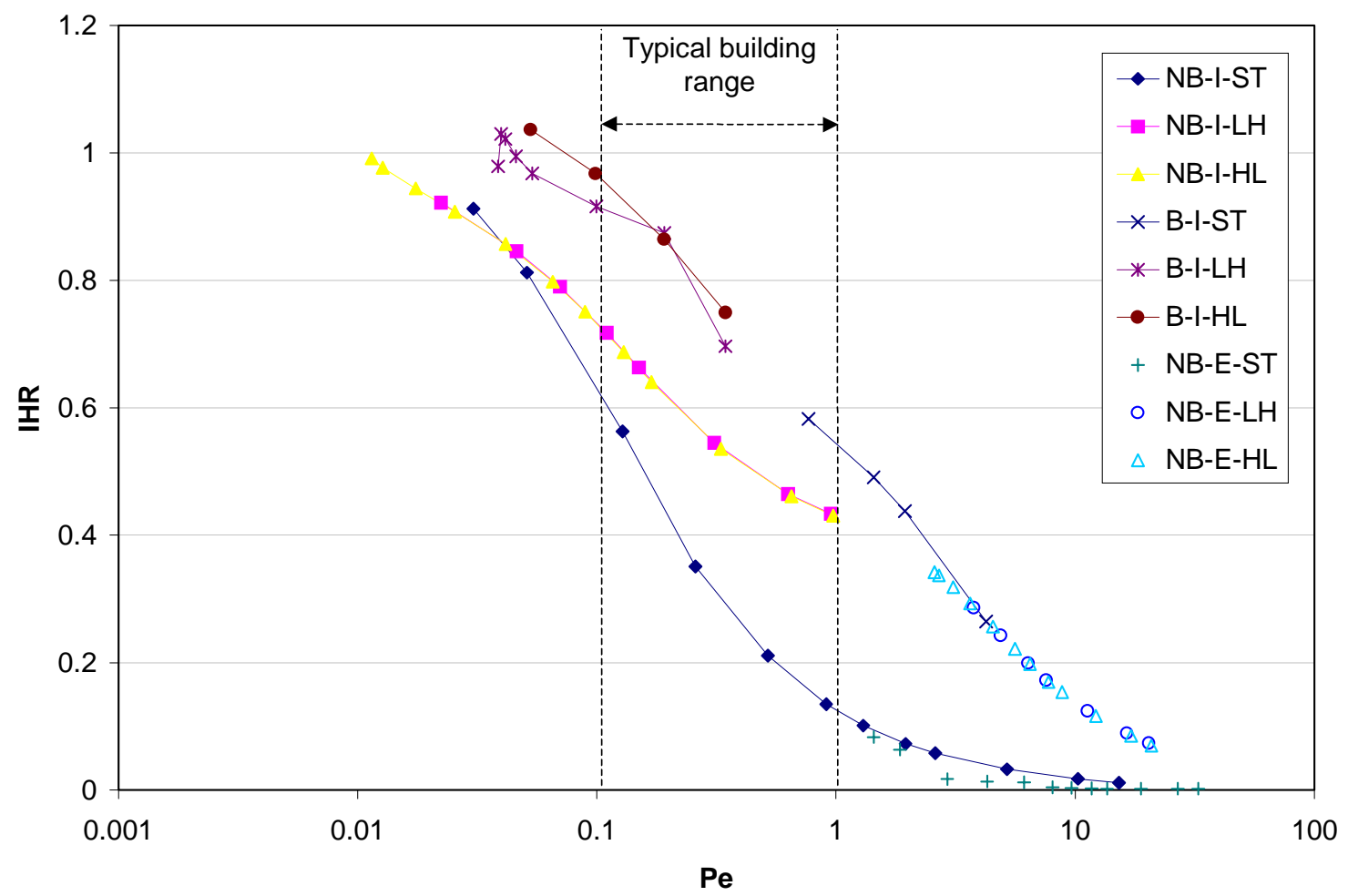

Figure 15: IHR as a function of Peclet number.

(Notes: NB: No air Boundary layers (temperature imposed directly on wall surfaces), B: air Boundary layers are included in the simulation, I: Insulated wall, E: Empty wall, ST:

Straight Through crack, LH: Low/High crack, HL: High/Low crack.)

Note that for the no boundary cases (NB), trends for insulated and empty walls look similar. The NB-I-LH/NB-I-HL and NB-E-LH/NB-E-HL curves are similar and the 
empty wall results appear to be an extension of the insulated wall results for higher Peclet number. For vertical walls, IHR occurs not only via the direct heat exchange between the leaking air-stream and conducting insulation within the wall, but also significantly via the participation of boundary layers on the outdoor and indoor wall surfaces in the infiltration or exfiltration.

In Figure 15, the IHR values for boundary layer cases seem to follow a single curve, independent of crack locations (i.e., at extremes of the wall or straight through). The IHR values for straight through cracks appear as a continuation of the low/high leakage results - just at higher Peclet number.

\subsubsection{IHR as a function of infiltrated air mass flux}

For almost all building engineers, the Peclet number of a building wall is somewhat non-intuitive concept. So, we present our IHR results in more familiar terms. Figure 16 shows IHR results as a function of air mass flux (kg/s per meter of wall length). Common values for air mass flux range from 0.0001 to $0.001 \mathrm{~kg} / \mathrm{s} . \mathrm{m}$. Trends displayed in Figure 16 appear similar to those in Figure 15, for dependence of IHR results on the Peclet number.

The difference between IHR results for NB-I-LH/HL and B-I-LH/HL is slightly smaller than in the previous graph, and the empty cavity results are shifted away from those for insulated walls due to the higher mass fluxes in the former.

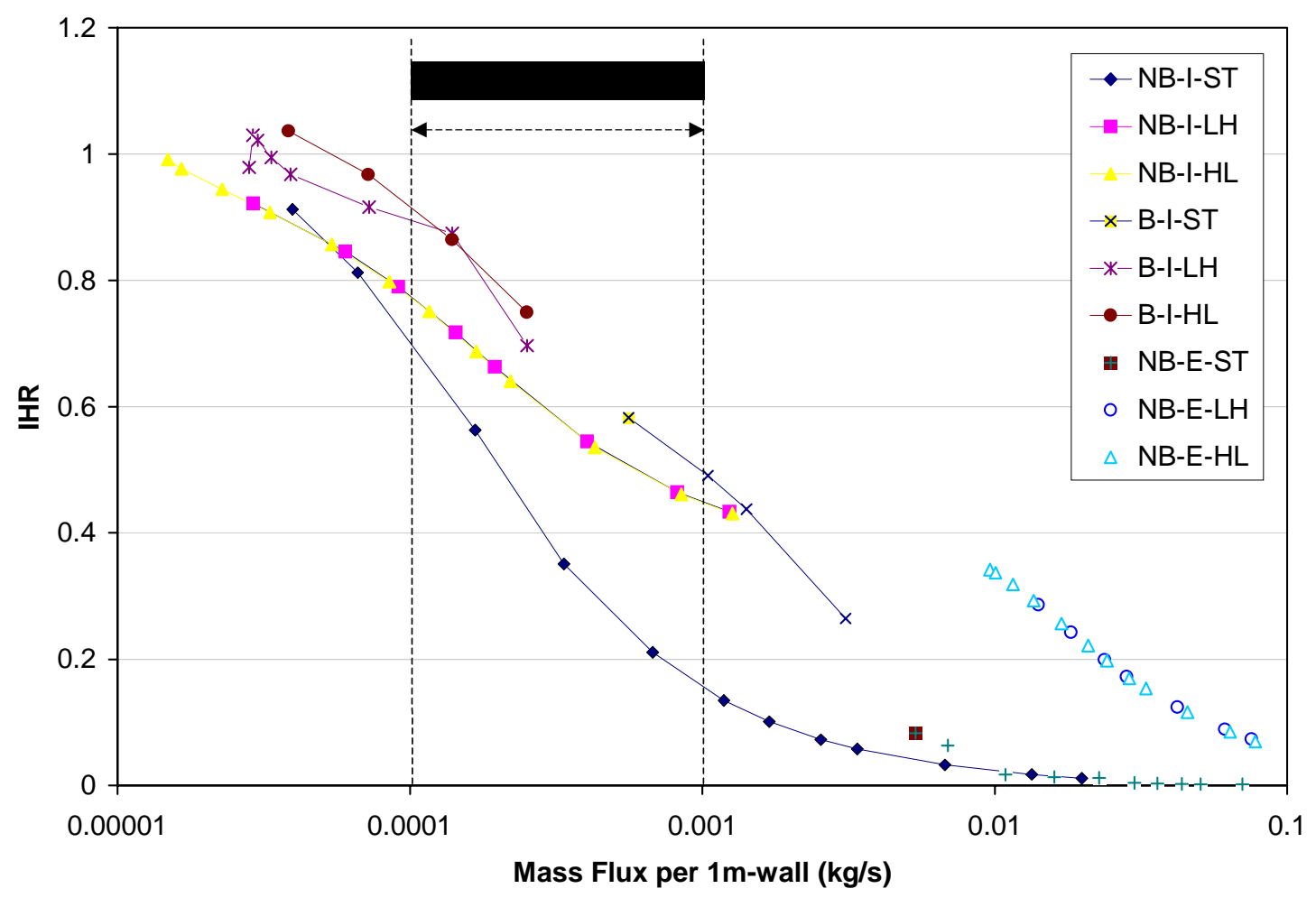

Figure 16: IHR as a function of infiltrated air mass flux per meter of wall. (Notes: NB: No air Boundary layers (temperature imposed directly on wall surfaces), B: air Boundary layers are included in the simulation, I: Insulated wall, E: Empty wall, ST: Straight Through crack, LH: Low/High crack, HL: High/Low crack.) 
6.1.3. IHR as a function of pressure difference across the wall

We now examine IHR values as a function of pressure difference across the wall. This has the advantage of viewing results for the same environmental conditions (wind and stack induced pressures). Typical indoor/outdoor pressure differences for residential buildings are within the range $0.1-10 \mathrm{~Pa}$.

The results shown in Figure 17 show that IHR values for low/high (LH) and high/low (HL) configurations are always greater than the ST configuration. Considering a typical pressure difference for a building for the no boundary layer (NB) case: IHR effect is zero for E-ST configuration, within $0.05-0.35$ for I-ST, between $0.15-0.35$ for E$\mathrm{LH} / \mathrm{HL}$, and has its highest values for the I-LH/HL (0.65-0.9). Infiltration heat recovery is most significant for insulated walls with long air flow path lengths (I-LH/HL walls) within the walls.

As in the previous discussions, the external air boundary layer effect is more important for the I-ST than for I-LH/HL, and IHR values increase when external boundary layer effects are taken into account. IHR values reach 0.6 for I-ST and 1.0 for ILH/HL.

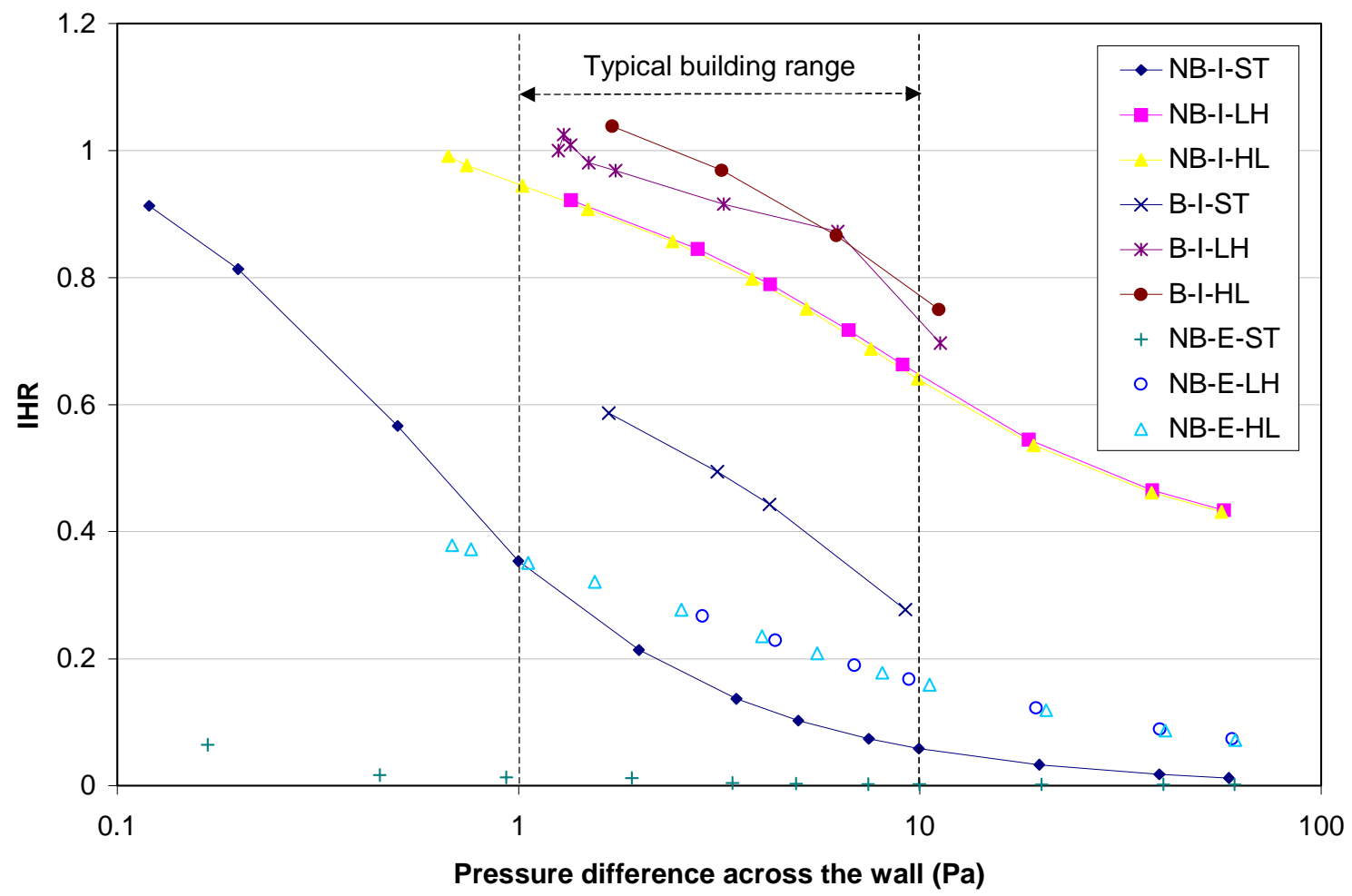

Figure 17: IHR as a function of pressure difference across the wall. (Notes: NB: No air Boundary layers (temperature imposed directly on wall surfaces), B: air Boundary layers are included in the simulation, I: Insulated wall, E: Empty wall, ST: Straight Through crack, LH: Low/High crack, HL: High/Low crack.) 


\subsection{Combining One Infiltrating and Three Exfiltrating Walls to Obtain a whole building IRH value}

Figure 18 presents a plan view of the second studied case. In this case, outdoor air infiltrates into the building through the upwind wall (wall 1) and leaves it through the three other walls (wall 2, 3 and 4).

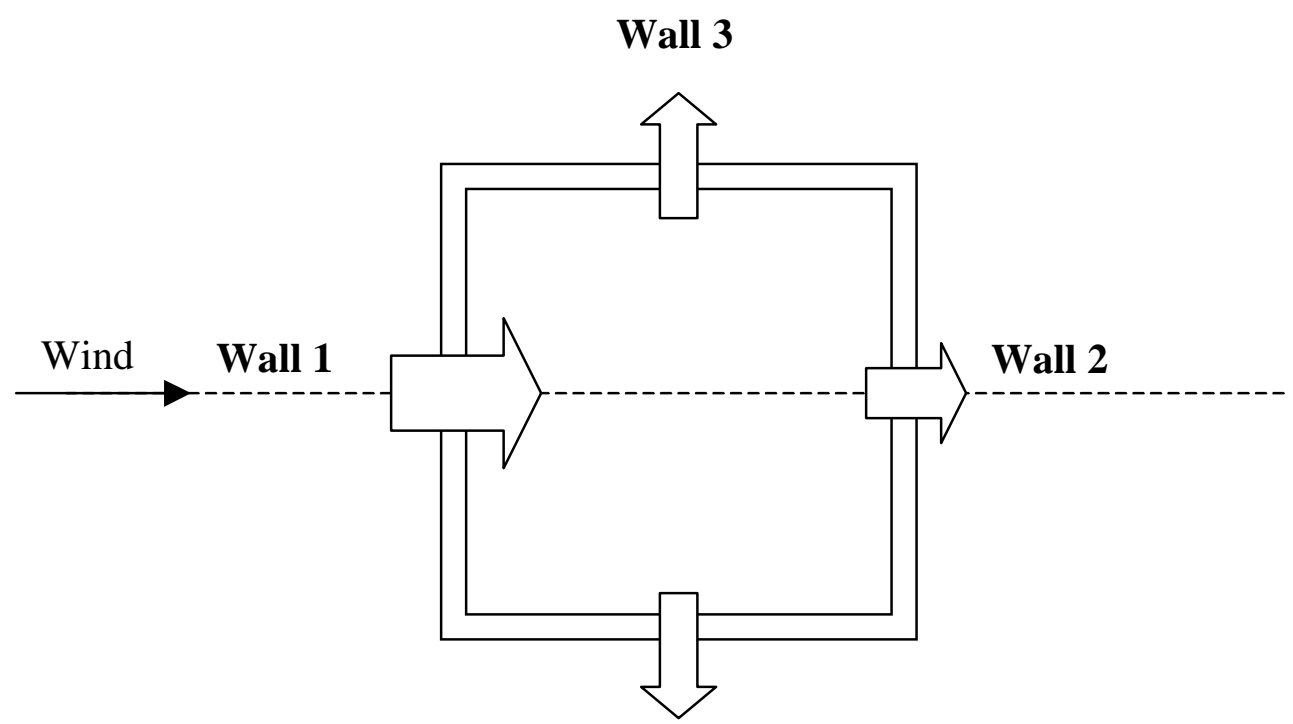

Wall 4

Figure 18: Case 2 - plan view.

For one infiltrating and three exfiltrating walls equation (9) leads to:

$$
I H R^{\text {Building }}=I H R_{1}^{I N F}+\frac{\sum_{j=2}^{4} P e_{j}^{E X F} \times I H R_{j}^{E X F}}{\sum_{j=2}^{4} P e_{j}^{E X F}}
$$

where $I H R^{I N F}, I H R^{E X F}$ and $I H R^{\text {Building }}$ are respectively the infiltration heat recovery factors for infiltrating and exfiltrating walls and building respectively and $P e$ is the wall Peclet number.

According to ASHRAE (2001) pressure coefficients for exfiltrating walls (walls 2, 3 and 4) are the same, therefore their air flows will be equal and the air mass flux through these walls is one third of that of the air mass flux through the infiltrating wall (wall 1). Equation (36) gives the resulting relation between Peclet numbers for infiltrating and exfiltrating walls.

$$
P e_{3}^{E X F}=P e_{4}^{E X F}=P e_{2}^{E X F}=\frac{1}{3} P e_{1}^{I N F}
$$


As previously, IHR for a building is calculated by combining the IHR effects for all infiltrating and exfiltrating wall sections. Linear interpolation permits us to estimate IHR for Peclet number between calculated values.

Figure 19 presents the IHR results as a function of Peclet number. On the whole, curves show the same trends than the previous case (Figure 15). It's more difficult to make comments on the boundary case (B) results because of the small number of points (only one for the B-I-ST and two for B-I-LH and B-I-HL).

Note that IHR values are slightly higher than those of the previous case, especially for the lowest Peclet numbers. This is the due to the higher participation of the exfiltrating walls whose IHR values are higher than those for infiltrating walls. We present the IHR results as a function of Peclet number only because of they are similar to the previous results (Figures 16 and 17).

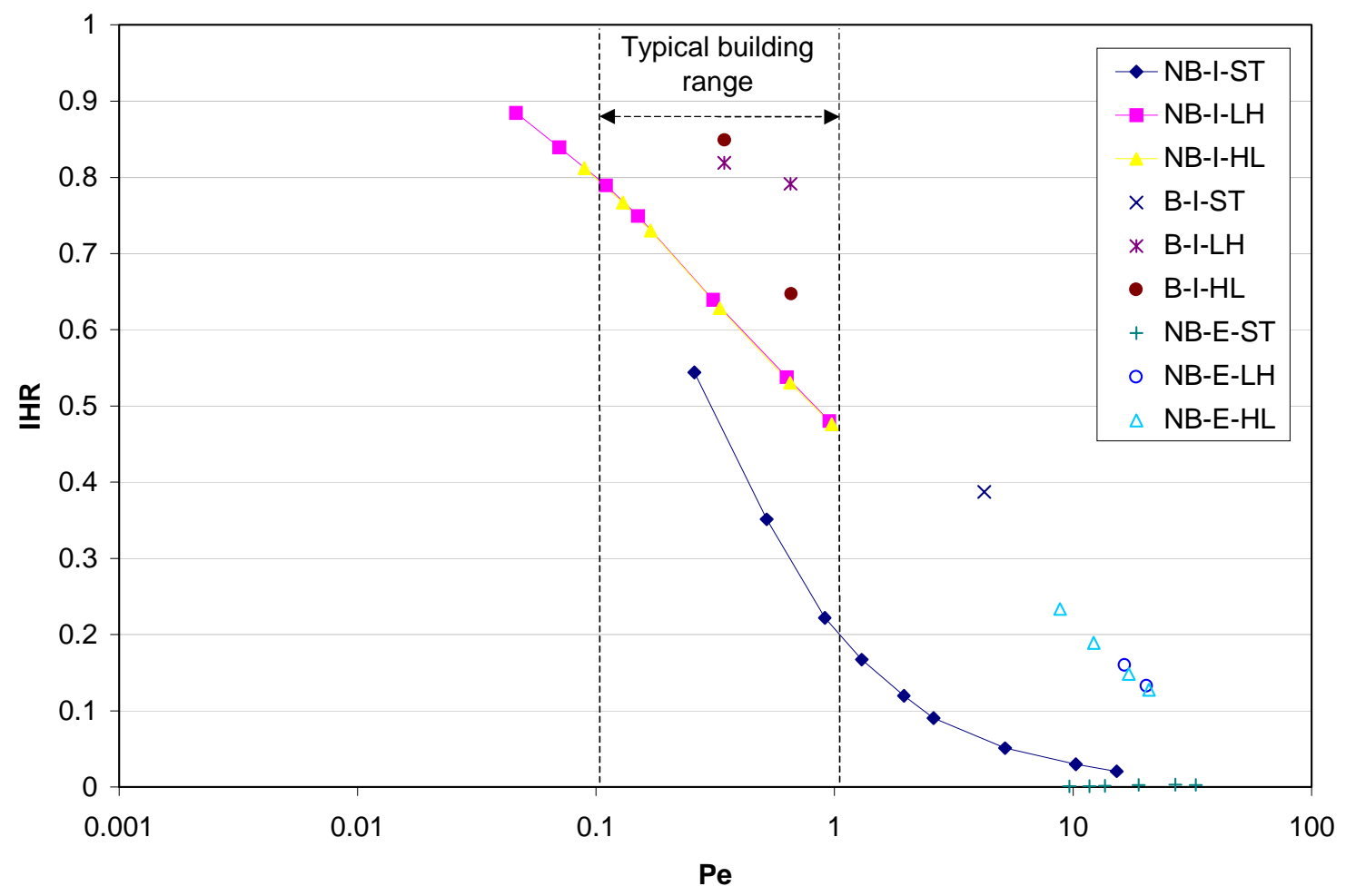

Figure 19: IHR as a function of Peclet number.

(Notes: NB: No air Boundary layers (temperature imposed directly on wall surfaces), B: air Boundary layers are included in the simulation, I: Insulated wall, E: Empty wall, ST: Straight Through crack, LH: Low/High crack, HL: High/Low crack.) 


\subsection{Comparison between the conventional and actual total energy load}

Figure 20 presents the percent difference between the two different estimates of conduction and infiltration heat load. The conventional estimate uses the classical equation (1), the improved estimate uses equation (3). The percent difference is shown as a function of Peclet number. The percent difference indicates the error that would be incurred in building energy load predictions that do not include infiltration heat recovery.

The percent error in conduction and infiltration heat load resulting from the use of the conventional calculational method (equation (1)) for this particular case is between $3 \%$ and $13 \%$ for Peclet numbers typically found in buildings. This confirms previous estimates made by Buchanan and Sherman (2000).

As previously, there are not enough calculational results to draw detailed conclusions. However, from the observations that all the boundary layer cases seem to follow a single curve independent of crack position, and that the difference between the NB and B curves remain the same (as shown in paragraph 5.1.1.), we conclude that a maximum value for percent error exists. This maximum percent error would be located at a Peclet number between 2 and 3 (out of the typical building range), and would be equal to about $20 \%$.

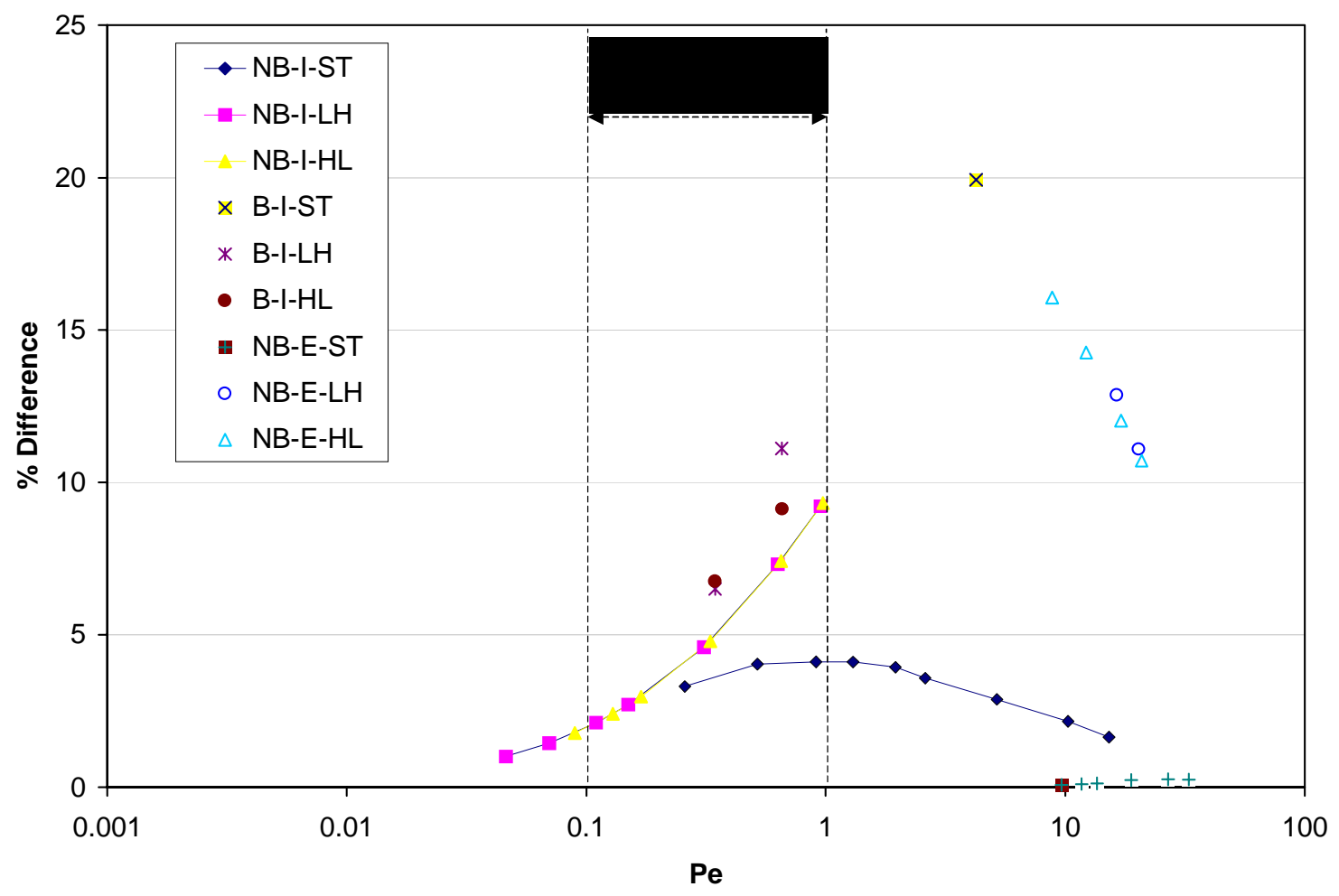

Figure 20: Percent difference between the conventional and actual total energy load.

(Notes: NB: No air Boundary layers (temperature imposed directly on wall surfaces), B: air Boundary layers are included in the simulation, I: Insulated wall, E: Empty wall, ST: Straight Through crack, LH: Low/High crack, HL: High/Low crack.) 


\section{Conclusion}

By passing through the building envelope, infiltrating air substantially modifies heat transfers through walls. When warm air flows out of a wall into a cold environment, it will warm the wall it is passing through, thus reducing conduction loss through it. The conventional calculation of building heat losses does not undertake this potential of heat recovery. The Infiltration Heat Recovery (IHR) factor was proposed by Sherman et al $(2000,2001)$ to quantify the heat recovery and to correct the conventional calculations.

Computational Fluid Dynamics (CFD) was used to calculate the infiltration heat recovery factor; specifically to understand factors that influence it, and assess its significance in building heat load calculations. CFD convergence issues cause problems for very low flows, but give reliable results for flows typically found in buildings.

Results show that the interior details of the wall encountered in the leakage path (i.e., insulated or empty walls) do not greatly influence the heat recovery. The overall crack geometry (i.e., inlet and outlet locations) is the key factor. This study shows for the first time the important effect of the external boundary layers on conduction and infiltration heat loads. Finally, our calculations suggest that the relative error in heat load resulting from the use of the conventional calculational method is between $3 \%$ and $13 \%$ for flows typically found in buildings.

\section{Acknowledgements}

The authors thank Max Sherman and Iain Walker from the Energy and Performance of Building Group for many thoughtful discussions.

\section{References}

ASHRAE Fundamentals. 2001. Airflow Around Buildings, p. 16.5, ASHRAE, Atlanta, CA.

Buchanan, C.R. and Sherman, M.H. 2000. A Mathematical Model for Infiltration Heat Recovery. LBNL 44294.

Caffey, G. E. 1979. Residential air infiltration. ASHRAE Trans., Vol. 85, pp. 41-57.

NIST. 1996. NIST estimates nationwide energy impact of air leakage in U. S. buildings. J. Research of NIST, Vol. 101, No. 3, p. 413.

Persily, A.1982. Understanding air infiltration in homes. Report PU/CEES No. 129, Princeton University Center for Energy and Environmental Studies, February, p. 335.

Sherman, M., Matson, N.1993. Ventilation-Energy liabilities in U.S. dwellings. Proc. $14^{\text {th }}$ AVIC Conference: Energy impact of ventilation and air infiltration, Copenhagen, September 21-23, pp. 23-41. Air Infiltration and Ventilation Centre, Coventry U.K.. Sherman, M.H. and Walker, I.S. 2001. Heat Recovery in Building Envelopes. Proc. ASHRAE/DOE/BTECC Conference on the Thermal Performance of the Exterior Envelopes of Buildings VIII. ASHRAE, Atlanta, GA. LBNL 47329.

STAR-CD Methodology. 1999. Computational Dynamics Limited, Version 3.1. 


\section{Appendix 1: Worked Example}

In this appendix, calculations are performed to illustrate the impact of the Infiltration Heat Recovery on building energy load predictions.

\section{Problem Description}

A plan view of a house is presented in Figure 18. This house has four identical walls of length $10 \mathrm{~m}$. The walls are made of wood frame construction, with a cavity created by having a layer of sheathing (plywood) on each side of vertical wooden studs. The plywood panels' width is $1.0 \mathrm{~cm}$ and thermal conductivity is $0.13 \mathrm{~W} / \mathrm{mK}$. The cavity is filled with insulation with thermal conductivity $0.041 \mathrm{~W} / \mathrm{mK}$. Walls are $2.44 \mathrm{~m}$ high and $10 \mathrm{~cm}$ thick. To simplify this example, air leaks and heat loss by conduction through the roof and floor are set to zero. The crack configuration chosen for this example is the straight through configuration. Also, for simplicity, we set heat conduction through the studs to be same as that through the insulation.

Indoor temperature is $23^{\circ} \mathrm{C}$ and outdoor temperature is $8^{\circ} \mathrm{C}$. Incident wind is normal to the infiltration wall. The normalized leakage is $0.75 \mathrm{ACH}$ (i.e. 0.75 volume of the house per hour), typical of US residential houses.

The goal of this example is to determine the heat losses through the house's envelope (vertical walls only) using both the conventional and the IHR-modified (i.e. taking the heat recovery into account) methods.

\section{Calculations}

- Conventional calculation of the heat loss by conduction trough the envelope

The heat loss by conduction is given by: $\quad \boldsymbol{Q}_{o}=\boldsymbol{U A} \times\left(\boldsymbol{T}_{\text {in }}-\boldsymbol{T}_{\text {out }}\right)$

- The $U$-value is equal to the inverse of the resistance, $R: \quad U=\frac{1}{R}=\frac{1}{\sum_{i=1}^{2} \frac{e_{i}}{\lambda_{i}}+R_{\text {film }}}$

where $e_{i}$ and $\lambda_{i}$ are the envelope constituent's width and thermal conductivity (sheathing and insulation), $R_{\text {film }}$ is the heat resistance of the air boundary layers on the external vertical sides of the walls (classically equal to $0.2 \mathrm{~m}^{2} . \mathrm{K} / \mathrm{W}$ ).

Then, the U-value is given by $\boldsymbol{U}=\frac{1}{\frac{0.01+0.01}{0.13}+\frac{0.08}{0.041}+0.2}=\mathbf{0 . 4 3} \mathbf{~ W} / \mathbf{m}^{2} . \mathbf{K}$

- The total surface area is $\boldsymbol{A}=(10.0 \times 2.44) \times 4$ walls $=\mathbf{9 7 . 6 0} \mathbf{~ m}^{2}$

- The total heat loss by conduction through the vertical walls is:

$$
\boldsymbol{Q}_{0}=0.43 \times 97.60 \times(23-15)=\mathbf{6 3 5 . 1 2} \mathrm{W}
$$


- Conventional calculation of the heat loss by infiltration trough the envelope

The heat loss by infiltration is given by: $\quad \boldsymbol{Q}_{I}=\dot{\boldsymbol{m}} \boldsymbol{c}_{p}\left(\boldsymbol{T}_{\text {in }}-\boldsymbol{T}_{\text {out }}\right)$

- The infiltration air mass flux is determined by: $\quad \dot{m}=\frac{A C H \times V}{\rho}$

where the volume of the house is $V=(10.0 \times 4) \times 2.44=97.60 \mathrm{~m}^{3}$ and the air density, $\rho$, is equal to $1.20 \mathrm{~kg} / \mathrm{m}^{3}$.

The air mass flux is then $\dot{\boldsymbol{m}}=\frac{0.75 \times 97.60}{1.20}=61.00 \mathrm{~kg} / \mathrm{h}=\mathbf{0 . 0 1 7} \mathbf{~ k g} / \mathbf{s}$

- The air heat capacity is $c_{p}=\mathbf{1 0 0 6} \mathbf{J} / \mathbf{K g} . \mathbf{K}$

- The heat loss by infiltration through the vertical walls is:

$$
Q_{I}=0.017 \times 1006 \times(23-15)=\mathbf{2 5 6 . 5 3} \mathbf{W}
$$

- Determination of the Infiltration Heat Recovery factors

The Infiltration Heat Recovery factor for the whole house is evaluated using equation (8) of the main text:

$$
I H R^{\text {House }}=I H R^{I N F}+\frac{\sum_{j=1}^{n_{E X F}} P e_{j}^{E X F} \times I H R_{j}^{E X F}}{\sum_{j=1}^{n_{E X F}} P e_{j}^{E X F}}
$$

In the present example, the walls are identical and the air mass fluxes through the

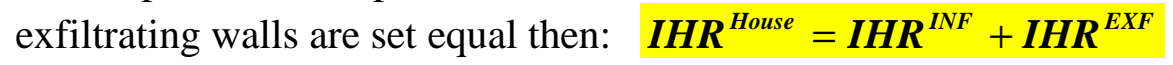

The Infiltration Heat Recovery factors for individual walls are determined using Figure 9 by calculating the required Peclet numbers.

$$
\begin{aligned}
& P e^{I N F}=\frac{\dot{m} c_{p}}{U A}=\frac{0.017 \times 1006}{0.43 \times(2.44 \times 10)}=1.63 \\
& P e_{1}^{E X F}=P e_{2}^{E X F}=P e_{3}^{E X F}=\frac{1}{3} P e^{I N F}=0.54
\end{aligned}
$$

The IHR factor for the whole house is $\mathbf{I H R ^ { \text { House } }}=0.22+0.32=\mathbf{0 . 5 4}$

- Impact of the heat recovery through the envelope

- The conventional calculation is given by equation (1) of the main text:

$$
Q_{C}=Q_{0}+Q_{I}=635.12+256.53=\mathbf{8 9 1 . 6 5 ~ W}
$$

- The IHR-modified calculation is given by equation (3) of the main text:

$$
\boldsymbol{Q}_{I H R}=Q_{0}+\left(1-I H R^{\text {House }}\right) \times Q_{I}=635.12+(1-0.54) \times 256.53=\mathbf{7 5 3 . 1 2} \mathbf{~ W}
$$

The impact of the infiltration heat recovery on the total heat loss through the verticals walls is $891.65-753.12=\mathbf{1 3 8 . 5 3} \mathbf{W}$. The heat recovery represents $\frac{138.53}{891.65}=\mathbf{1 5 . 5 \%}$ of the conventional calculated heat loss. 